\title{
Relativistic mean-field interaction with density-dependent meson-nucleon vertices based on microscopical calculations
}

\author{
X. Roca-Maza, ${ }^{1,2, *}$ X. Viñas, ${ }^{1, \dagger}$ M. Centelles, ${ }^{1, \ddagger}$ P. Ring,,${ }^{3, \S}$ and P. Schuck ${ }^{4,5,6, \|}$ \\ ${ }^{1}$ Departament d'Estructura i Constituents de la Matèria and Institut de Ciències del Cosmos, Facultat de Física, \\ Universitat de Barcelona, Diagonal 647, E-08028 Barcelona, Spain \\ ${ }^{2}$ INFN, sezione di Milano, via Celoria 16, I-20133 Milano, Italy \\ ${ }^{3}$ Physikdepartment, Technische Universität München, D-85748 Garching, Germany \\ ${ }^{4}$ Institut de Physique Nucléaire, CNRS, UMR8608, F-91406 Orsay, France \\ ${ }^{5}$ Université Paris-Sud, F-91505 Orsay, France \\ ${ }^{6}$ Laboratoire de Physique et Modélisation des Milieux Condensés, F-38042 Grenoble, France
}

(Received 17 August 2011; published 14 November 2011)

\begin{abstract}
Although $a b$ initio calculations of relativistic Brueckner theory lead to large scalar isovector fields in nuclear matter, at present, successful versions of covariant density functional theory neglect the interactions in this channel. A new high-precision density functional DD-ME $\delta$ is presented which includes four mesons, $\sigma, \omega, \delta$, and $\rho$, with density-dependent meson-nucleon couplings. It is based to a large extent on microscopic ab initio calculations in nuclear matter. Only four of its parameters are determined by adjusting to binding energies and charge radii of finite nuclei. The other parameters, in particular the density dependence of the meson-nucleon vertices, are adjusted to nonrelativistic and relativistic Brueckner calculations of symmetric and asymmetric nuclear matter. The isovector effective mass $m_{p}^{*}-m_{n}^{*}$ derived from relativistic Brueckner theory is used to determine the coupling strength of the $\delta$ meson and its density dependence.
\end{abstract}

DOI: 10.1103/PhysRevC.84.054309

PACS number(s): 21.60.Jz, 21.30.Fe, 21.60.De, 21.65.Ef

\section{INTRODUCTION}

Structure properties of nuclei described in the framework of effective mean-field interactions are remarkably successful over almost the entire periodic table [1-12]. Relativistic and nonrelativistic versions of this approach enable an effective description of the nuclear many-body problem as an energy density functional. These energy functionals are usually adjusted to a variety of finite nuclei and infinite nuclear matter properties. Although all these effective interactions are based on the mean-field approach, some differences will generally appear among them due to the specific ansatz of the density dependence adopted for each interaction. For instance, predictions in the isovector channel of existing functionals differ widely from one another and, as a consequence, the density dependence of the symmetry energy is far from being fully determined. This has an impact on finite nuclei properties such as, for example, the neutron skin thickness. Mean-field models, which accurately describe the charge radius in ${ }^{208} \mathrm{~Pb}$, predict neutron radii between 6.6 and $5.8 \mathrm{fm}$. For these reasons, one of the main goals in nuclear physics is to build a universal density functional theory based on microscopic calculations $[13,14]$. This functional should be able to explain as many as possible measured data within the same parameter set and to provide reliable predictions for properties of nuclei far from stability not yet or never accessible to experiments in the laboratory. It should be derived in a fully microscopic

\footnotetext{
*roca@ecm.ub.es

${ }^{\dagger}$ xavier@ecm.ub.es

${ }^{\ddagger}$ mario@ecm.ub.es

§ring@ph.tum.de

" schuck@ipno.in2p3.fr
}

way from the interactions between bare nucleons. At present, however, attempts to derive such a density functional provide only qualitative results for two reasons: first, the three-body term of the bare interaction is not known well enough and, second, the methods to derive such a functional are not precise enough to achieve the required accuracy. Note that a $1 \%$ error in the binding energy per particle of symmetric nuclear matter leads to an error of several $\mathrm{MeV}$ in the binding energy of heavy nuclei, an error which is an order of magnitude larger than required by astrophysical applications. Therefore, at present the most successful functionals are derived either in a fully phenomenological way from a very large set of experimental data, as for instance more than 2000 nuclear binding energies [8], or, more recently, by an adjustment to a combination of microscopic results and to a set of characteristic experimental data [15-18].

With the same goal, in the more recent past a different approach was tried. Baldo, Schuck, and Viñas employed the same route as in condensed matter physics and constructed a functional (BCP) [12] where the bulk part is based on the fully microscopic calculations of Ref. [19]. In this more fundamental calculation, Baldo and collaborators investigate the nuclear and infinite neutron matter on the basis of the Bethe-Brueckner approach including three-body correlations of the Bethe-Faddeev type [20]. Their results for the equation of state (EoS) of symmetric and neutron matter are believed to be among the most accurate in the literature. Then, the BCP functional took as a benchmark the calculations of Ref. [19] by means of a polynomial fit. In this way an accurate and analytic EoS as a function of neutron and proton densities was constructed covering the whole range from symmetric nuclear matter to pure neutron matter in density ranges from zero to about twice saturation density. Subsequently, a 
finite-range surface term dependent on three parameters together with the strength of the spin-orbit term (four parameters in total) was added to the bulk part of the functional. The pairing correlations needed to describe open-shell nuclei were accounted for by a density-dependent $\delta$ force with an effective mass equal to the nucleon mass that simulates pairing calculations in symmetric nuclear matter computed with the Gogny interaction [21] in the $T=1$ channel. Adjusting these free parameters to some selected nuclear experimental data yielded excellent results for nuclear masses and reasonable charge radii of the whole nuclear chart. In addition it has also been shown that the deformation properties of $\mathrm{BCP}$ functionals are similar to the ones found using the Gogny D1S force [22,23] in spite of the fact that both models are clearly different. A recent review on the BCP functionals can be found in Ref. [24]

In general, symmetries of nature help to reduce the number of parameters and to simplify the description. One of the underlying symmetries of QCD is Lorentz invariance and therefore covariant versions of density functionals are of particular interest in nuclear physics. This symmetry allows one to describe the spin-orbit coupling, which has an essential influence on the underlying shell structure in finite nuclei, in a consistent way. Moreover, it also puts stringent restrictions on the number of parameters in the corresponding functionals without reducing the quality of the agreement with experimental data. Self-consistent mean-field calculations starting from relativistic Lagrangians have been very successful in describing nuclear properties [25-29]. They arise from a microscopic treatment of the nuclear many-body problem in terms of nucleons and mesons carrying the effective interaction between nucleons. Moreover, since the theory is relativistically invariant and the field and nucleon equations of motion are solved self-consistently, they preserve causality and provide a self-consistent description of the spin-orbit term of the nuclear effective force and of the bulk and surface parts of the interaction. In addition, these functionals include nuclear magnetism [30], i.e., a consistent description of currents and time-odd fields, important for odd-mass nuclei [31], excitations with unsaturated spins, magnetic moments [32], and nuclear rotations [33]. No new parameters are required for the time-odd parts of the mean fields. In nonrelativistic functionals the corresponding time-odd parts are usually difficult to adjust to experimental data and even if there are additional constraints derived from Galilean invariance and gauge symmetry [34] these constraints are usually not taken into account in the successful functionals commonly used in the literature. The earlier versions of covariant density functional theory were based on the Walecka model [35-38] with phenomenological nonlinear meson interactions proposed by Boguta and Bodmer [39], introducing in this way a phenomenological density dependence $[4,9,40]$. Later the nonlinear models have been replaced by an explicit density dependence of the meson-nucleon vertices. This density dependence has first been determined in a phenomenological way $[6,7,10]$. These models have shown considerable improvements with respect to previous relativistic mean-field (RMF) models in the description of asymmetric nuclear matter, neutron matter, and nuclei far from the stability valley. On the other hand, attempts have been made to derive this density dependence in a microscopic way from Brueckner calculations in nuclear matter at various densities [41-44]. An example is density-dependent relativistic hadron field theory [42] in which the specific density dependence of the meson-nucleon vertices is mapped from Dirac-Brueckner calculations where the in-medium interaction is obtained from nucleon-nucleon potentials consistent with scattering experiments. Therefore, if this ansatz is adopted, the effective theory is derived fully from first-principles calculations. Of course, the accuracy of the results obtained in this way is by no means satisfactory for modern nuclear structure calculations and a fit of additional free parameters is still needed. This fact allows us to constrain the different possibilities and keeps the compatibility, at least theoretically, with more fundamental calculations of infinite nuclear matter.

As mentioned, there exist $a b$ initio calculations of the nuclear EoS over a wide range of nucleon densities, i.e., far from densities currently reachable in the laboratory. In this sense, apart from the experimental data needed in the fitting procedure for determining an effective interaction, further steps in building a universal density functional may need to implement such $a b$ initio information, as was done in the BCP model. Therefore, this EoS calculated from first principles can be understood as a temporary benchmark at supra- and subsaturation densities of the energy per particle at different asymmetries. Furthermore, from a theoretical point of view, consistency is desirable between predictions of both theories. Regrettably, to make them compatible is not only a problem of including the EoS derived from realistic nucleon-nucleon potentials within the fitting procedure. It is also a problem of taking into account the proper density dependence in the different terms of the functional. For that, first-principles calculations are also thought to be the best candidate to help in building a universal energy density functional, at least the bulk part of it since many-nucleon calculations are not feasible yet. Hence, for an effective and self-consistent treatment of the nuclear many-body problem, we propose here an improved relativistic mean-field interaction with an explicit density dependence of the meson-nucleon vertices in all four spin-isospin channels compatible with fully microscopic calculations.

The essential breakthrough of density functional methods in the description of quantum mechanical many-body problems was Kohn-Sham theory $[45,46]$, where the exact density functional $E[\rho]$ of Hohenberg and Kohn [47] was mapped in an exact way on an effective potential $V_{\text {eff }}$ in a single-particle Schroedinger equation, which forms the starting point of all modern applications of density functional theory. In covariant density functional theory this effective potential corresponds to the self-energy in the Dirac equation, which can be decomposed into four channels characterized by the relativistic quantum numbers of spin and isospin, the scalar isoscalar channel $(J=0, T=0)$, the vector isoscalar channel $(J=1, T=$ $0)$, the scalar isovector channel $(J=0, T=1)$, and the vector isovector channel $(J=1, T=1)$. In the Walecka model these channels are connected with the exchange of mesons carrying the corresponding quantum numbers. However, in nearly all the present successful phenomenological applications of covariant density functional theory to nuclear structure based on the relativistic Hartree model only the three mesons $\sigma, \omega$, 
and $\rho$ are taken into account. The scalar isovector $\delta$ meson $\left[a_{0}(980)\right]$ causing a splitting of the effective mass between protons and neutrons is neglected, because it has turned out that usual data such as binding energies and radii of finite nuclei do not allow us to distinguish scalar and vector fields in the isovector channels. Allowing independent parameters for the $\rho$ and the $\delta$ mesons leads to redundancies in the fit. For the same reasons modern relativistic Hartree-Fock [48-50] and HartreeFock-Bogoliubov [51] calculations also neglect the $\delta$ meson in the Lagrangian and, as a consequence, in the direct term. Of course, the Fock term of these calculations also contains contributions to the scalar isovector channel. Microscopic investigations by Huber et al. [52,53] and phenomenological studies [54-64] in the literature stressed that mean-field models which neglect the $\delta$ meson are likely to miss important ingredients in describing properly very asymmetric nuclear matter, in particular at high densities. The proton fraction of $\beta$-stable matter in neutron stars can increase and the splitting of the effective mass can affect transport properties in neutron stars and heavy-ion reactions. However, as long as the parameters of this meson are not fixed, such investigations are somewhat academic. Therefore we derive in this paper the $\delta$-nucleon vertex and its density dependence from modern microscopic calculations based on the bare nucleon-nucleon force of the Tübingen group [65].

The relativistic mean-field model DD-ME $\delta$ obtained in this way is an extension of the DD-ME model developed by the Munich group $[7,10]$ based on density-dependent relativistic Hartree theory. The DD-ME model has the following degrees of freedom: the proton, the neutron, and three mesons carrying the nuclear interaction, namely, $\sigma, \omega$, and $\rho$ mesons. In addition to these degrees of freedom, we include here a new one, the $\delta$ meson, for the reasons pointed out before. Since this meson provides a treatment of the isospin more close to that of the microscopic investigations we can hope that it improves the reliability of the models for predictions in nuclei far from stability with large isospin, such as planned to be studied experimentally at the new Rare Ion Beam Facilities [66]. Apart from the inclusion of the $\delta$ meson in DD-ME $\delta$ the DD-ME $\delta$ model differs from the earlier DD-ME models in that the parameters of DD-ME were all adjusted to experimental data based on finite nuclei properties, whereas those of DD-ME $\delta$ are largely based on microscopic ab initio calculations in nuclear matter. Only four of the parameters of DD-ME $\delta$ are fitted to finite nuclei.

The paper is organized as follows: after establishing the DD-ME $\delta$ model in Sec. II, we discuss in Sec. III the strategies to determine the parameters of the Lagrangian and compare in Sec. IV the results of this effective interaction with the experiment and with the nonrelativistic BCP model [12] and the completely phenomenological DD-ME2 model [10], in particular focusing on binding energies, charge radii, and neutron skins in spherical nuclei.

\section{DENSITY-DEPENDENT HADRON FIELD THEORY}

\section{A. Lagrangian and equations of motion}

Density-dependent relativistic hadron field theory, which forms the basis of the DD-ME $\delta$ interaction, has been formulated and extensively discussed in Refs. [42,67,68]. Here we present only the essential features of the mean-field equations of motion derived from such a theory. The relativistic Lagrangian includes neutrons and protons represented by the Dirac spinors $\psi$ of the nucleon, the four mesons $(\sigma, \omega, \delta$, and $\rho$ ) carrying the effective nuclear strong interaction represented by the fields $\sigma, \omega^{\mu}, \vec{\delta}$, and $\vec{\rho}^{\mu}$, and the photon field $A^{\mu}$ accounting for the electromagnetic interaction. The index $\mu$ indicates the time- and space-like components of the vector fields and the arrow indicates the vector nature of a field in isospin space. As mentioned, the $\delta$ meson should be included if one wants to follow the theoretical indications of Dirac-Brueckner calculations in asymmetric nuclear matter and so we do. The Lagrangian has the following parts:

$$
\mathcal{L}=\mathcal{L}_{N}+\mathcal{L}_{M}+\mathcal{L}_{\text {int }},
$$

where $\mathcal{L}_{N}$ is the nucleonic free Lagrangian,

$$
\mathcal{L}_{N}=\bar{\psi}\left(i \gamma_{\mu} \partial^{\mu}-m\right) \psi,
$$

$\mathcal{L}_{M}$ is the Lagrangian of free mesons,

$$
\begin{aligned}
\mathcal{L}_{M}= & \frac{1}{2}\left(\partial_{\mu} \sigma \partial^{\mu} \sigma-m_{\sigma}^{2} \sigma^{2}\right)+\frac{1}{2}\left(\partial_{\mu} \vec{\delta} \partial^{\mu} \vec{\delta}-m_{\sigma}^{2} \vec{\delta}^{2}\right) \\
& -\frac{1}{4} \Omega_{\mu \nu} \Omega^{\mu \nu}-\frac{1}{2} m_{\omega}^{2} \omega_{\mu} \omega^{\mu}-\frac{1}{4} \vec{R}_{\mu \nu} \vec{R}^{\mu \nu}-\frac{1}{2} m_{\rho}^{2} \vec{\rho}_{\mu} \vec{\rho}^{\mu} \\
& -\frac{1}{4} F_{\mu \nu} F^{\mu \nu}
\end{aligned}
$$

and $\mathcal{L}_{\text {int }}$ is the Lagrangian describing the interactions. Its algebraic expression is

$$
\begin{aligned}
\mathcal{L}_{\text {int }}= & g_{\sigma} \bar{\psi} \sigma \psi+g_{\delta} \bar{\psi} \vec{\tau} \vec{\delta} \psi \\
& -g_{\omega} \bar{\psi} \gamma_{\mu} \omega^{\mu} \psi-g_{\rho} \bar{\psi} \gamma_{\mu} \vec{\tau} \vec{\rho}^{\mu} \psi-e \bar{\psi} \gamma_{\mu} A^{\mu} \psi,
\end{aligned}
$$

where $m$ is the nucleon mass (commonly taken as $939 \mathrm{MeV}$ ); the field strength tensors for the vector fields are

$$
\Omega^{\mu v}=\partial^{\mu} \omega^{v}-\partial^{v} \omega^{\mu}
$$

and correspondingly $\vec{R}^{\mu \nu}$ and $F^{\mu \nu}$. The electric charge is $e$ for protons and zero for neutrons. The meson-nucleon vertices are denoted by $g_{i}$ for $i=\sigma, \omega, \delta$, and $\rho$. Since covariance is required and the quantity $\sqrt{j_{\mu} j^{\mu}}$ is in the rest frame identical to the baryon density $\rho=\rho_{n}+\rho_{p}$, the nucleon-meson vertices generally depend on this quantity. Because of the relatively small velocities the difference between $\sqrt{j_{\mu} j^{\mu}}$ and $\rho$ is negligible in all practical applications. The subindex $n$ or $p$ is used to indicate whether we are considering neutrons or protons, respectively.

The equations of motion are derived from the classical variational principle and we obtain for the nucleon spinors the Dirac equation

$$
\left[\gamma_{\mu}\left(i \partial^{\mu}-\Sigma^{\mu}\right)-m^{*}\right] \psi=0,
$$

where $m^{*} \equiv m-\Sigma^{s}$ is the effective Dirac nucleon mass and $\Sigma^{\mu}$ and $\Sigma^{s}$ are the vector and scalar self-energies defined as follows:

$$
\begin{aligned}
\Sigma^{s} & \equiv g_{\sigma}(\rho) \sigma+g_{\delta}(\rho) \vec{\tau} \vec{\delta}, \\
\Sigma^{\mu} & \equiv \Sigma^{(0) \mu}+\delta_{\mu 0} \Sigma^{(r)} .
\end{aligned}
$$


Here, (0) indicates the usual definition of the vector self-energy and $(r)$ the rearrangement term of the vector self-energy:

$$
\begin{aligned}
\Sigma^{(0) \mu} & \equiv g_{\omega}(\rho) \omega^{\mu}+g_{\rho}(\rho) \tau_{3} \rho_{3}^{\mu}+e A^{\mu}, \\
\Sigma^{(r)} & \equiv-\frac{d g_{\sigma}}{d \rho} \sigma \rho^{s}+\frac{d g_{\omega}}{d \rho} \omega^{0} \rho-\frac{d g_{\delta}}{d \rho} \delta_{3} \rho_{3}^{s}+\frac{d g_{\rho}}{d \rho} \rho_{3}^{0} \rho_{3} .
\end{aligned}
$$

Here $e A^{0}$ is the direct term of the Coulomb potential. As in most RMF models we neglect in these investigations the Coulomb exchange term, which plays an important role in pn random-phase approximation calculations [69]. The static mean-field approximation used throughout this investigation preserves the third component of the isospin. As a consequence the other two components of the densities and fields carrying isospin vanish. In Eq. (9) and the following equations $\rho_{3}^{0}$ represents the time-like component of the $\rho$-meson field, whereas $\rho_{3}=\rho_{n}-\rho_{p}$ and $\rho_{3}^{s}=\rho_{n}^{s}-\rho_{p}^{s}$ represent the isovector part of the baryon density and of the scalar density. The rearrangement term is a contribution to the vector self-energy due to the density dependence of the meson-nucleon vertices. The equations of motion for the mesons are

$$
\begin{aligned}
\left(\partial_{\nu} \partial^{\nu}+m_{\sigma}^{2}\right) \sigma & =-g_{\sigma}(\rho) \rho^{s}, \\
\left(\partial_{\nu} \partial^{\nu}+m_{\omega}^{2}\right) \omega^{\mu} & =+g_{\omega}(\rho) j^{\mu}, \\
\left(\partial_{\nu} \partial^{\nu}+m_{\delta}^{2}\right) \delta_{3} & =-g_{\delta}(\rho) \rho_{3}^{s}, \\
\left(\partial_{\nu} \partial^{\nu}+m_{\rho}^{2}\right) \rho_{3}^{\mu} & =+g_{\rho}(\rho) j_{3}^{\mu}, \\
\partial_{\nu} \partial^{\nu} A^{\mu} & =+e j_{p}^{\mu},
\end{aligned}
$$

where the different densities and currents are the ground-state expectation values defined as

$$
\begin{aligned}
\rho^{s} & \equiv\langle 0|\bar{\psi} \psi| 0\rangle=\rho_{n}^{s}+\rho_{p}^{s}, \\
j^{\mu} & \equiv\left\langle 0\left|\bar{\psi} \gamma^{\mu} \psi\right| 0\right\rangle=j_{n}^{\mu}+j_{p}^{\mu}, \\
\rho_{3}^{s} & \equiv\left\langle 0\left|\bar{\psi} \tau_{3} \psi\right| 0\right\rangle=\rho_{n}^{s}-\rho_{p}^{s}, \\
j_{3}^{\mu} & \equiv\left\langle 0\left|\bar{\psi} \gamma^{\mu} \tau_{3} \psi\right| 0\right\rangle=j_{n}^{\mu}-j_{p}^{\mu} .
\end{aligned}
$$

\section{B. Asymmetric infinite nuclear matter}

\section{Energy density and pressure}

In infinite nuclear matter we neglect the electromagnetic field. Because of translational invariance, the Dirac equations can be solved analytically in momentum space and we obtain the usual plane-wave Dirac spinors [70]. Filling up to the Fermi momenta $k_{F \tau}$ for $\tau=n$ or $p$, we find the densities

$$
\begin{aligned}
\rho_{\tau} & =\frac{2}{(2 \pi)^{3}} \int_{|k|<k_{F \tau}} d^{3} k=\frac{k_{F \tau}^{3}}{3 \pi^{2}}, \\
\rho_{\tau}^{s} & =\frac{2}{(2 \pi)^{3}} \int_{|k|<k_{F \tau}} \frac{m_{\tau}^{*}}{E_{\tau}(k)} d^{3} k \\
& =\frac{m_{\tau}^{*}}{2 \pi^{2}}\left[k_{F \tau} E_{F \tau}-m_{\tau}^{* 2} \ln \left(\frac{k_{F_{\tau}}+E_{F \tau}}{m_{\tau}^{*}}\right)\right]
\end{aligned}
$$

and the meson fields

$$
\sigma=-\frac{g_{\sigma}(\rho)}{m_{\sigma}^{2}}\left(\rho_{n}^{s}+\rho_{p}^{s}\right)
$$

$$
\begin{gathered}
\omega^{0}=+\frac{g_{\omega}(\rho)}{m_{\omega}^{2}}\left(\rho_{n}+\rho_{p}\right), \\
\delta_{3}=-\frac{g_{\delta}(\rho)}{m_{\delta}^{2}}\left(\rho_{n}^{s}-\rho_{p}^{s}\right), \\
\rho_{3}^{0}=+\frac{g_{\rho}(\rho)}{m_{\rho}^{2}}\left(\rho_{n}-\rho_{p}\right),
\end{gathered}
$$

where $E_{\tau}(k)=\sqrt{\mathbf{k}^{2}+m_{\tau}^{* 2}}$ and where the Fermi energy of neutrons and protons is given by $E_{F \tau}=E_{\tau}\left(k_{F \tau}\right)$. Now, we calculate the energy density $(\epsilon)$ and pressure $(P)$ from the energy-momentum tensor

$$
T^{\mu \nu}=\sum_{i} \frac{\partial \mathcal{L}}{\partial\left(\partial_{\mu} \phi_{i}\right)} \partial^{v} \phi_{i}-g^{\mu \nu} \mathcal{L},
$$

where $\phi_{i}$ runs over all possible fields,

$$
\begin{aligned}
\epsilon= & \left\langle 0\left|T^{00}\right| 0\right\rangle \\
= & \frac{1}{4}\left[3 E_{F n} \rho_{n}+m_{n}^{*} \rho_{n}^{s}\right]+\frac{1}{4}\left[3 E_{F p} \rho_{p}+m_{p}^{*} \rho_{p}^{s}\right] \\
& +\frac{1}{2}\left[m_{\sigma}^{2} \sigma^{2}+m_{\omega}^{2}\left(\omega^{0}\right)^{2}+m_{\delta}^{2} \delta_{3}^{2}+m_{\rho}^{2}\left(\rho_{3}^{0}\right)^{2}\right]
\end{aligned}
$$

and

$$
\begin{aligned}
P= & \frac{1}{3} \sum_{i=1}^{3}\left\langle 0\left|T^{i i}\right| 0\right\rangle \\
= & \frac{1}{4}\left[E_{F n} \rho_{n}-m_{n}^{*} \rho_{n}^{s}\right]+\frac{1}{4}\left[E_{F p} \rho_{p}-m_{p}^{*} \rho_{p}^{s}\right] \\
& -\frac{1}{2}\left[m_{\sigma}^{2} \sigma^{2}-m_{\omega}^{2}\left(\omega^{0}\right)^{2}+m_{\delta}^{2} \delta_{3}^{2}-m_{\rho}^{2}\left(\rho_{3}^{0}\right)^{2}\right] \\
& +\left(\rho_{n}+\rho_{p}\right) \Sigma^{(r) 0} .
\end{aligned}
$$

Only the pressure has a rearrangement contribution. We have checked that the pressure derived from the energy-momentum tensor coincides with the thermodynamical definition of $p=\rho^{2}[\partial(\epsilon / \rho) / \partial \rho]$ and that the energy-momentum tensor is conserved: $\partial_{\mu} T^{\mu \nu}=0$.

\section{The symmetry energy: $S_{2}(\rho)$}

By assuming charge symmetry for the strong interaction (and noting that the $n n$ and $p p$ interactions are identical but different, in general, from the $n p$ interaction), the total energy per particle in asymmetric nuclear matter can be written as follows:

$$
\frac{\epsilon}{\rho}=\frac{E}{A} \equiv e(\rho, \alpha)=e(\rho, \alpha=0)+S_{2}(\rho) \alpha^{2}+O\left[\alpha^{4}\right],
$$

where $\rho=\rho_{n}+\rho_{p}$ is the baryon density and $\alpha=\left(\rho_{n}-\right.$ $\left.\rho_{p}\right) /\left(\rho_{n}+\rho_{p}\right)$ measures the neutron excess. The term proportional to $\alpha^{2}$ is the so-called symmetry energy of infinite matter and terms proportional to $\alpha^{4}$ (and higher) can be neglected to very good approximation. The symmetry energy $S_{2}(\rho)$ is defined as

$$
S_{2}(\rho)=\frac{1}{2}\left(\frac{\partial^{2} e(\rho, \alpha)}{\partial \alpha^{2}}\right)_{\alpha=0}
$$


Models including the $\delta$ meson provide a richer description of the isovector sector of the nuclear strong interaction. For that reason it is important to understand its effects on asymmetric nuclear matter and for that we give the analytic expressions for the symmetry energy of the model discussed in the last section [56,57]:

$$
S_{2}(\rho) \equiv S_{2}^{\mathrm{kin}}(\rho)+S_{2}^{\rho}(\rho)+S_{2}^{\delta}(\rho),
$$

with

$$
\begin{gathered}
S_{2}^{\mathrm{kin}}(\rho)=\frac{k_{F}^{2}}{6 E_{F}}, \\
S_{2}^{\rho}(\rho)=\frac{1}{2} \rho \frac{g_{\rho}^{2}}{m_{\rho}^{2}}, \\
S_{2}^{\delta}(\rho)=-\frac{1}{2} \rho \frac{g_{\delta}^{2}}{m_{\delta}^{2}}\left(\frac{m^{*}}{E_{F}}\right)^{2} u_{\delta}\left(\rho, m^{*}\right),
\end{gathered}
$$

where for $i=\sigma, \delta$

$$
u_{i}\left(\rho, m^{*}\right) \equiv \frac{1}{1+3 \frac{g_{i}^{2}}{m_{i}^{2}}\left(\frac{\rho^{s}}{m^{*}}-\frac{\rho}{E_{F}}\right)} .
$$

The quantity $u_{\sigma}\left(\rho, m^{*}\right)$ will be needed below. In these equations we used the fact that for $\alpha=0$ we have $\rho_{n}=$ $\rho_{p}=\rho / 2, \rho_{n}^{s}=\rho_{p}^{s}=\rho^{s} / 2, \rho_{3}^{s}=0$, and $m_{n}^{*}=m_{p}^{*}$ and we have defined $k_{F}^{3}=3 \pi^{2} \rho / 2$ and $E_{F}=\sqrt{k_{F}^{2}+m^{* 2}}$. In symmetric nuclear matter, the effective mass and the scalar density read

$$
\begin{aligned}
& m^{*}=m-\frac{g_{\sigma}^{2}}{m_{\sigma}^{2}} \rho^{s}, \\
& \rho^{s}=\frac{m^{*}}{\pi^{2}}\left[k_{F} E_{F}-m^{* 2} \ln \left(\frac{k_{F}+E_{F}}{m^{*}}\right)\right],
\end{aligned}
$$

respectively. Close to the saturation density, $u_{\delta} \approx 1$ is a very good approximation and we find in this case the analytical approximation

$$
S_{2}^{\delta}(\rho) \approx-\frac{1}{2} \rho \frac{g_{\delta}^{2}}{m_{\delta}^{2}}\left(\frac{m^{*}}{E_{F}}\right)^{2},
$$

and, therefore, the contribution to the symmetry energy coming from the nuclear strong interaction (potential part) as described by this kind of model can be written in the simple form

$$
\begin{aligned}
S_{2}^{\text {pot }}(\rho) & =S_{2}^{\rho}(\rho)+S_{2}^{\delta}(\rho) \\
& \approx \frac{1}{2} \rho\left[\frac{g_{\rho}^{2}}{m_{\rho}^{2}}-\frac{g_{\delta}^{2}}{m_{\delta}^{2}}\left(\frac{m^{*}}{E_{F}}\right)^{2}\right] .
\end{aligned}
$$

The symmetry energy is often expanded around the saturation density $\rho_{\text {sat }}$ as

$$
S_{2}(\rho)=J+\frac{L}{3 \rho_{\text {sat }}}\left(\rho-\rho_{\text {sat }}\right)+\frac{K_{\text {sym }}}{18 \rho_{\text {sat }}^{2}}\left(\rho-\rho_{\text {sat }}\right)^{2}+\ldots,
$$

where $J$ is the symmetry energy at saturation, and $L$ and $K_{\text {sym }}$ are proportional, respectively, to the slope and the curvature of the symmetry energy at saturation.
Using the analytical expressions (33) we find

$$
L(\rho) \equiv 3 \rho \frac{d S_{2}(\rho)}{d \rho}=L^{\mathrm{kin}}(\rho)+L^{\rho}(\rho)+L^{\delta}(\rho),
$$

with

$$
\begin{aligned}
L^{\mathrm{kin}}(\rho)= & S_{2}^{\mathrm{kin}}\left(2-\frac{k_{F}^{2}}{E_{F}^{2}}-\frac{3 m^{* 2}}{E_{F}^{2}} w\right), \\
L^{\rho}(\rho)= & S_{2}^{\rho}\left(3+6 \frac{\rho}{g_{\rho}} \frac{\partial g_{\rho}}{\partial \rho}\right), \\
L^{\delta}(\rho)= & S_{2}^{\delta}\left[3+6 \frac{\rho}{g_{\delta}} \frac{\partial g_{\delta}}{\partial \rho}-\frac{2 k_{F}^{2}}{E_{F}^{2}}+6\left(1-\frac{m^{* 2}}{E_{F}^{2}}\right) w\right. \\
& -3 \frac{g_{\delta}^{2}}{m_{\delta}^{2}} u_{\delta}\left(2 v\left(\frac{\rho}{g_{\delta}} \frac{\partial g_{\delta}}{\partial \rho}+w\right)\right. \\
& \left.\left.+\rho \frac{k_{F}^{2}}{E_{F}^{3}}(1-3 w)\right)\right],
\end{aligned}
$$

where the functions $u_{i}, v$, and $w$ depend on $\rho$ and $m^{*}$ :

$$
\begin{aligned}
v\left(\rho, m^{*}\right) & \equiv 3\left(\frac{\rho^{s}}{m^{*}}-\frac{\rho}{E_{F}}\right), \\
w\left(\rho, m^{*}\right) & \equiv \frac{\rho}{m^{*}} \frac{\partial m^{*}}{\partial \rho} \\
& =-\frac{g_{\sigma}^{2}}{m_{\sigma}^{2}} u_{\sigma}\left(2 \frac{\rho^{s}}{m^{*}} \frac{\rho}{g_{\sigma}} \frac{\partial g_{\sigma}}{\partial \rho}+\frac{\rho}{E_{F}}\right) .
\end{aligned}
$$

The strength of the $\sigma$ - and $\omega$-nucleon vertices is quite well determined by experimental data as compared with the strength of the isovector meson-nucleon vertices. On the other side, with only the $\rho$-nucleon vertex, one is able to reproduce properties of finite nuclei [71] and to account for the symmetry energy around saturation in rather good agreement with available empirical indications. However, to reproduce nucleon-nucleon scattering measurements in vacuum, one needs to incorporate a scalar-isovector meson into the parametrization of the two-body nuclear interaction [72]. Microscopic derivations of the nuclear fields using relativistic Brueckner theory $[42,52,53,65,73-75]$ or nonrelativistic Brueckner theory $[43,44]$ show clearly that the scalar field in the nuclear interior has an isovector part. These reasons motivate one to incorporate the $\delta$ meson also in models of covariant density functional theory and to study its influence on properties such as the symmetry energy, the effective mass splitting between protons and neutrons in asymmetric matter, the isospin dependence of the spin-orbit potential, and the spin-orbit splittings far from stability.

\section{Density dependence of the meson-nucleon vertices}

Here we describe the density dependence of the mesonnucleon vertices used for the new interaction DD-ME $\delta$. We start from modern fully microscopic calculations in symmetric nuclear matter and pure neutron matter at various densities and try to determine the density dependence of the vertices $g_{i}(\rho)$ by fitting to those data. Of course, it is well known that successful density functionals can, at present, not be determined completely from ab initio calculations. Therefore, we introduce in the fit not only results of microscopic 
calculations but also a set of data on binding energies and radii in specific finite nuclei.

In a first step we have to choose a form of the density dependence of the various vertices which is flexible enough to reproduce the microscopic calculations. In Refs. [41,76] the meson-nucleon vertices of density-dependent RMF theory have been related to the scalar and vector self-energies obtained from Dirac-Brueckner (DB) calculations in infinite nuclear matter. The density dependence deduced from DB calculations is

$$
g_{i}(\rho)=g_{i}\left(\rho_{\text {sat }}\right) f_{i}(x) \text { for } i=\sigma, \omega, \delta, \rho,
$$

where $\rho_{\text {sat }}$ is the saturation density of symmetric nuclear matter and $x=\rho / \rho_{\text {sat }}$. For the functions $f_{i}(x)$ we follow Refs. $[6,7,10]$ and use the Typel-Wolter ansatz

$$
f_{i}(x)=a_{i} \frac{1+b_{i}\left(x+d_{i}\right)^{2}}{1+c_{i}\left(x+e_{i}\right)^{2}} .
$$

As in Refs. [7,10,16] we use the value $\rho_{\text {sat }}=0.152 \mathrm{fm}^{-3}$. In fact, this choice is very close to the saturation density obtained in the following fit. As we see from the ansatz (46) the actual value of $\rho_{\text {sat }}$ is irrelevant for the calculations. It can be completely absorbed in the values of the parameters $b_{i}, c_{i}$, $d_{i}$, and $e_{i}$. It is only used to make them dimensionless. By definition, the parameters $a_{i}$ are constrained by the condition $f(1)=1$. In earlier applications $[6,7,10]$ this ansatz was only used for the $\sigma$ and $\omega$ meson. The density dependence of the isovector coupling $g_{\rho}(\rho)$ was described by an exponential and the $\delta$ meson was neglected. Here we use the same ansatz (46) also for the isovector mesons $\delta$ and $\rho$. This turned out to be necessary in order to obtain a density dependence of the $\delta$ coupling similar to that derived from microscopic ab initio calculations in Refs. [42,76,77]. We impose as in Ref. [6] the constraints $e_{\sigma}=d_{\sigma}, e_{\omega}=d_{\omega}, f_{\sigma}^{\prime \prime}(x=1)=f_{\omega}^{\prime \prime}(x=1)$, and $f_{i}^{\prime \prime}(x=0)=0$. We work with meson masses $m_{\omega}=783 \mathrm{MeV}$, $m_{\delta}=983 \mathrm{MeV}$, and $m_{\rho}=763 \mathrm{MeV}$. The nucleon mass is $m=$ $939 \mathrm{MeV}$. All in all, the model has 14 adjustable parameters: the 4 coupling constants $g_{i}\left(\rho_{\text {sat }}\right)$ in the four relativistic channels (Lorentz-scalar, Lorentz-vector, isoscalar and isovector), 9 parameters describing the density dependence in the functions $f_{i}(x)$, and the $\sigma$ mass $m_{\sigma}$ allowing for a finite range and a proper description of the nuclear surface.

\section{Calculation of finite nuclei}

The self-consistent results for masses include a microscopic estimate for the center-of-mass correction:

$$
E_{\mathrm{cm}}=-\frac{\left\langle P_{\mathrm{cm}}^{2}\right\rangle}{2 m A}
$$

where $P_{\mathrm{cm}}$ is the total momentum of a nucleus with $A$ nucleons. The expression

$$
r_{\mathrm{c}}=\sqrt{\left\langle r_{p}^{2}\right\rangle+0.8^{2}}
$$

is used for the charge radius. The description of open shell nuclei requires pairing correlations. We introduce this through the BCS approach with a seniority zero force in the soft pairing window described in Ref. [78]. For the fit of the parameters of the Lagrangian described in the next section the constant-gap approximation [79] has been used and the gap parameters have been derived from the experimental binding energies by a three-point formula.

\section{THE PARAMETERS OF THE FUNCTIONAL DD-ME $\delta$}

In this section we describe the determination of the parameters of DD-ME $\delta$. Earlier fits of relativistic Lagrangians have shown that the usual set of experimental ground-state properties in finite nuclei, such as binding energies and radii, do not allow us to determine more than seven or eight parameters [4]. Two of them $\left(g_{\sigma} / m_{\sigma}\right.$ and $\left.g_{\omega} / m_{\omega}\right)$ determine the saturation energy and the saturation density of symmetric nuclear matter [37], one of them $\left(m_{\sigma}\right)$ is fixed by the radii in finite nuclei, and another $\left(g_{\rho} / m_{\rho}\right)$ determines the symmetry energy $J$ at saturation. The additional parameters (such as, for instance, $g_{2}$ and $g_{3}$ in the nonlinear meson coupling models NL1 [3] or NL3 [4] or the three parameters in the ansatz (46) for density dependence in the isoscalar channel of DD-ME1 [7] and DD-ME2 [10]) are determined by the isoscalar surface properties and are necessary to describe deformations and the nuclear incompressibility properly. Finally, one parameter $\left(a_{\rho}\right.$ in DD-ME1 or DD-ME2) is needed to describe the density dependence of the symmetry energy by a fit to the experimental data on the neutron skin thickness.

In order to calibrate the 14 free parameters of the DD-ME $\delta$ functional we therefore added pseudo-data in the form of results of modern microscopic nonrelativistic and relativistic Brueckner calculations. To this end, we selected the EoS of symmetric nuclear matter and of neutron matter (see Fig. 1) derived by Baldo et al. [19] in a state-of-the-art nonrelativistic Brueckner calculation including relativistic corrections and three-body forces. We also used as a benchmark the isovector part of the effective Dirac mass $m_{p}^{*}-m_{n}^{*}$ (see Fig. 2) derived by the Tübingen group [65] in relativistic Dirac-Brueckner theory. The use of nonrelativistic results for the EoS and of relativistic results for the isovector effective mass may seem somewhat arbitrary. However, we have to keep in mind that the nonrelativistic calculations of the Catania group are more sophisticated than presently available Dirac-Brueckner

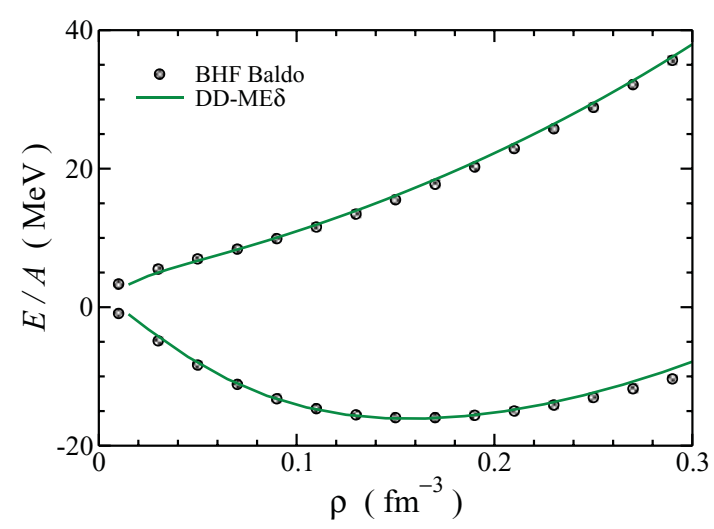

FIG. 1. (Color online) Equation of state for symmetric nuclear matter and for pure neutron matter as a function of the nucleon density. The dots represent the predictions of the BHF calculations [19] and the line our fit to reproduce these data. 


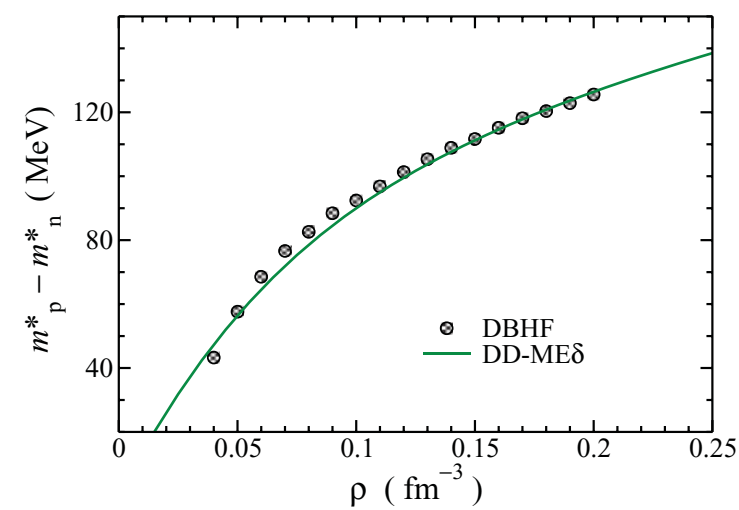

FIG. 2. (Color online) Proton-neutron effective mass splitting as a function of the nucleon density in pure neutron matter. The dots represent the predictions of the DBHF calculations [65] and the line our fit to reproduce these data.

calculations, because they include not only relativistic effects but also three-body forces. On the other hand, it is very complicated to deduce Dirac masses from a nonrelativistic calculation which does not distinguish between Lorentz scalars and vectors. This is in principle possible [43,44], but it is difficult and connected with additional uncertainties. With this caveat in mind, we decided to use a reliable relativistic Brueckner calculation [65] providing directly the effective Dirac masses $m_{p}^{*}$ and $m_{n}^{*}$ and their difference, a quantity directly connected with the scalar isovector part of the self-energy. The isovector part of the effective Dirac mass $m_{p}^{*}-m_{n}^{*}$ depends only on the $\delta$ meson. It vanishes for all the conventional Lagrangians without the $\delta$ meson. The density dependence of this quantity is therefore the optimal tool to get information about the density dependence of the $\delta$-meson vertex $g_{\delta}(\rho)$.

Keeping this in mind, we determine 10 of the 14 parameters in the Lagrangian of DD-ME $\delta$ by these pseudo-data obtained from ab initio calculations of nuclear matter. These parameters define the density dependence for the various meson-nucleon vertices (nine parameters) and the strength $g_{\delta}\left(\rho_{\text {sat }}\right)$ of the $\delta$ meson. Only a reduced set of four parameters $\left[g_{\sigma}\left(\rho_{\text {sat }}\right)\right.$, $g_{\omega}\left(\rho_{\text {sat }}\right), g_{\rho}\left(\rho_{\text {sat }}\right)$, and $\left.m_{\sigma}\right]$ is then fitted to the masses and charge radii of finite nuclei.

\section{A. Strategy of the parameter fit}

Since the mean-field equations of motion have to be solved self-consistently, we need a good starting parameter set before fixing the $\delta$-meson coupling to the above-mentioned calculations fully. The density-dependent meson coupling model DD-ME2 [10] provides us with an excellent description of nuclei all over the periodic table. Though DD-ME2 neglects the $\delta$ meson, it is based in the isoscalar channel on the same ansatz (46). Therefore, we used DD-ME2 as a starting point of our investigations. We proceeded in three steps:

(i) We performed an overall fit with all 14 parameters. For the data we have chosen on one side the three microscopic curves for the equations of state in Figs. 1 and 2 and on the other side the same set of data of finite nuclei which has been used in Ref. [10] for the determination of the parameter set DD-ME2 (see Table II of this reference), i.e., 12 binding energies of spherical nuclei distributed all over the periodic table and 9 charge radii. Because the density dependence in the isovector channel is determined by the equation of state of neutrons it was not necessary to include data on neutron skin thicknesses $\left(r_{n}-r_{p}\right)$. This fit provides us with a relatively stable starting point for a subsequent fine tuning of the model. Moreover, since the $\delta$ meson is little influenced by the overall fit to finite nuclei both $g_{\delta}\left(\rho_{\text {sat }}\right)$ and $f_{\delta}(x)$ (four parameters) are relatively well determined already in this step and we need only a fine tuning of the remaining parameters in the next two steps.

(ii) We keep the four meson masses and the four parameters describing the density-dependent vertex $g_{\delta}(\rho)$ of the $\delta$ meson fixed and determine the nine parameters describing the Typel-Wolter ansatz for the density-dependent vertices $g_{i}(\rho)$ of the remaining three mesons $(i=\sigma$, $\omega$, and $\rho$ ) by a very accurate fit to the nuclear matter data shown in Figs. 1 and 2. Because it involves only nuclear matter data, this is a relatively fast calculation and as a result we obtain the three density-dependent vertices $g_{i}(\rho)$ for $i=\sigma, \omega, \rho$. In this way we describe with high precision the equations of state for symmetric nuclear matter and pure neutron matter as well as the isovector part of the effective Dirac mass $\Delta m^{*}=$ $m_{p}^{*}-m_{n}^{*}$.

(iii) We keep the $\delta$-meson parameters as determined in step 1 and the density-dependent functions $f_{i}(x)$ for $i=\sigma, \omega, \rho$ are frozen at the values found in step 2 . We refine the remaining four parameters $g_{\sigma}\left(\rho_{\text {sat }}\right), g_{\omega}\left(\rho_{\text {sat }}\right)$, $g_{\rho}\left(\rho_{\text {sat }}\right)$, and $m_{\sigma}$ to the binding energies of 161 spherical nuclei and the charge radii of 86 nuclei shown in Table III, taking into account in this fit also the pseudodata of the nuclear matter properties used in steps 1 and 2 with certain weights. It turns out that the values of $g_{\sigma}\left(\rho_{\text {sat }}\right), g_{\omega}\left(\rho_{\text {sat }}\right), g_{\rho}\left(\rho_{\text {sat }}\right)$, and $m_{\sigma}$ obtained in this fit differ only slightly from the values determined in step 1 and that the nuclear matter results (the equations of state in symmetric nuclear matter and pure neutron matter as well as the isovector Dirac mass) differ only marginally from the results obtained in step 2 . As a consequence the procedure involving steps 2 and 3 does not have to be repeated.

The final parameter set DD-ME $\delta$ obtained in this way is given in Table I. It is compared with the parameter set DD-ME2 in Table II. We observe a large difference in the value of the the $\rho$-nucleon vertex $g_{\rho}$. This can be understood by the fact that we have in DD-ME2 only one meson $(\rho)$ in the isovector channel with a very different density dependence. We also observe a very different density dependence for the isoscalar mesons $\sigma$ and $\omega$. All in all, this has only a minor effect in the low-density region $\rho \leqslant \rho_{\text {sat }}$ but it has a large effect at high densities, as shown in Fig. 3. 


\section{B. $\chi^{2}$ definition}

Our fit is performed through a $\chi^{2}$ test of the form

$$
\chi^{2}=\frac{1}{n_{\text {data }}} \sum_{i=1}^{n_{\text {data }}} w_{i}^{2}\left(\mathcal{O}_{i}^{\text {model }}-\mathcal{O}_{i}^{\text {ref }}\right)^{2},
$$

where $n_{\text {data }}$ is the number of data points and $w_{i}$ the weight associated to each data point. $\mathcal{O}_{i}^{\text {ref }}$ is the experimental value for finite nuclei and the pseudo-data obtained by ab initio calculations in nuclear matter. The observables in finite nuclei used for the fit are the binding energies of 161 nuclei and the charge radii of 86 nuclei given in Table III. All of the isotopes are spherical even-even nuclei and the data are taken from the literature [80]. In the standard definition of a $\chi^{2}$ test, the weights $w_{i}$ should be inversely proportional to the experimental uncertainties. However, in the case of energies these are usually so small, that they cannot be used as relevant quantities. We therefore used the weights given in Table IV, i.e., $w_{i}=1 / 0.5 \mathrm{MeV}^{-1}$ for the masses and $w_{i}=1 / 0.01 \mathrm{fm}^{-1}$ for the radii. For the fit to the results of $a b$ initio calculations in nuclear matter we use $n_{\text {data }}$ mesh points in a certain density range (see Table IV) and we assume a relative accuracy of $3 \%$. The minimization of $\chi^{2}$ is carried out by means of a variable metric method algorithm included in the MINUIT package of Ref. [81].

In the first step of the fit discussed in the last section we minimize the quantity

$$
\chi_{(1)}^{2}=\chi_{B}^{2}+\chi_{r_{c}}^{2}+\chi_{\text {sym }}^{2}+\chi_{\text {neut }}^{2}+\chi_{\Delta \mathrm{m}^{*}}^{2}
$$

At this stage all 14 parameters of the model are varied and the data of finite nuclei are restricted to the masses and charge radii of the 12 nuclei used in the fit of the parameter set DD-ME2 in Ref. [10]. As we have described in the last section, the parameters for the $\delta$ meson obtained from this fit are no longer changed. We have given them in the third line of Table I.

In the second step we minimize

$$
\chi_{(2)}^{2}=\chi_{\text {sym }}^{2}+\chi_{\text {neut }}^{2}+\chi_{\Delta \mathrm{m}^{*}}^{2}
$$

for nuclear matter data with respect to the six constants characterizing the density dependence of the $\sigma, \omega$, and $\rho$ mesons and the three couplings $g_{\sigma}\left(\rho_{\text {sat }}\right), g_{\omega}\left(\rho_{\text {sat }}\right)$, and $g_{\rho}\left(\rho_{\text {sat }}\right)$. (Here, the $\delta$ meson and $m_{\sigma}$ are held to the same values found in the first step.) The obtained values for the constants $a_{i}, b_{i}, c_{i}, d_{i}, e_{i}$ that define the density dependence of the $i=\sigma, \omega, \rho$ meson-nucleon vertices are given in Table I.

In the third step we minimize $\chi^{2}$ for the nuclear matter data and the 161 binding energies and 86 charge radii given in

TABLE I. The parameter set DD-ME $\delta$ with the $\delta$ meson. It includes 14 independent parameters, only 4 of which $\left[g_{\sigma}\left(\rho_{\text {sat }}\right)\right.$, $g_{\omega}\left(\rho_{\text {sat }}\right), g_{\rho}\left(\rho_{\text {sat }}\right)$, and $\left.m_{\sigma}\right]$ are fitted to finite nuclei. The other 10 are derived from an adjustment to ab initio calculations in infinite nuclear matter $[19,65]$.

\begin{tabular}{lrrccccc}
\hline \hline$i$ & $m_{i}(\mathrm{MeV})$ & $g_{i}\left(\rho_{\mathrm{sat}}\right)$ & $a_{i}$ & $b_{i}$ & $c_{i}$ & $d_{i}$ & $e_{i}$ \\
\hline$\sigma$ & 566.1577 & 10.3325 & 1.3927 & 0.1901 & 0.3679 & 0.9519 & 0.9519 \\
$\omega$ & 783.0000 & 12.2904 & 1.4089 & 0.1698 & 0.3429 & 0.9860 & 0.9860 \\
$\delta$ & 983.0000 & 7.1520 & 1.5178 & 0.3262 & 0.6041 & 0.4257 & 0.5885 \\
$\rho$ & 763.0000 & 6.3128 & 1.8877 & 0.0651 & 0.3469 & 0.9417 & 0.9737 \\
\hline \hline
\end{tabular}

TABLE II. The parameter set DD-ME2, which does not contain a $\delta$ meson. It includes eight independent parameters, all of which are fitted to finite nuclei. In DD-ME2 the density dependence of the $\rho$ meson is given by $g_{\rho}(\rho)=g_{\rho}\left(\rho_{\text {sat }}\right) \exp \left[-a_{\rho}(x-1)\right]$.

\begin{tabular}{rrrccccc}
\hline \hline$i$ & $m_{i}(\mathrm{MeV})$ & $g_{i}\left(\rho_{\mathrm{sat}}\right)$ & $a_{i}$ & $b_{i}$ & $c_{i}$ & $d_{i}$ & $e_{i}$ \\
\hline$\sigma$ & 550.1238 & 10.5396 & 1.3881 & 1.0943 & 1.7057 & 0.4421 & 0.4421 \\
$\omega$ & 783.0000 & 13.0189 & 1.3892 & 0.9240 & 1.4620 & 0.4775 & 0.4775 \\
$\rho$ & 763.0000 & 3.6836 & 0.5647 & & & & \\
\hline \hline
\end{tabular}

Table III:

$$
\chi_{(3)}^{2}=\chi_{B}^{2}+\chi_{r_{c}}^{2}+\chi_{\text {sym }}^{2}+\chi_{\text {neut }}^{2}+\chi_{\Delta \mathrm{m}^{*}}^{2}
$$

Now we fit only a restricted set of four parameters, i.e., the three couplings $g_{\sigma}\left(\rho_{\text {sat }}\right), g_{\omega}\left(\rho_{\text {sat }}\right)$, and $g_{\rho}\left(\rho_{\text {sat }}\right)$ and $m_{\sigma}$. The resulting values are given in the first two columns of Table I.

We have to emphasize that only four free parameters$g_{\sigma}\left(\rho_{\text {sat }}\right), g_{\omega}\left(\rho_{\text {sat }}\right), g_{\rho}\left(\rho_{\text {sat }}\right)$ and $m_{\sigma}$-have been used in the final fit to the experimental data in finite nuclei. The other 10 parameters are derived from $a b$ initio calculations. This is in contrast to the typical relativistic and nonrelativistic fits of mean-field interactions, where commonly around 10 free parameters are adjusted to data in finite nuclei. It is also worth remembering that adding the $\delta$ meson has improved our theoretical picture of the nucleus and of the EoS of asymmetric nuclear matter.

So far we have used in the fit only nuclei with spherical shapes. The pairing correlations are treated in the first step in

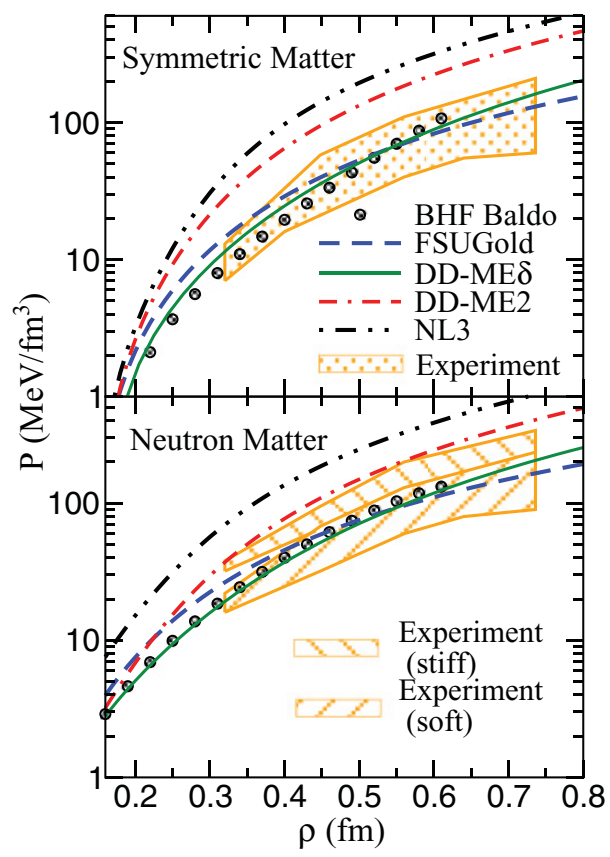

FIG. 3. (Color online) The pressure defined in Eq. (27) for symmetric nuclear matter (upper panel) and for neutron matter (lower panel) as a function of the density. The results for DD-ME $\delta$ are compared with those of the density functionals NL3 from Ref. [4], FSUGold from Ref. [9], DD-ME2 from Ref. [10], and microscopic BHF calculations from Ref. [19]. The shaded area represents experimental results from Ref. [83]. 
TABLE III. DD-ME $\delta$ results for the binding energies and charge radii used in the fit.

\begin{tabular}{ccccccccccccccc}
\hline \hline Elem. $N$ & $\begin{array}{c}B \\
(\mathrm{MeV})\end{array}$ & $\begin{array}{c}B^{\text {exp. }} \\
(\mathrm{MeV})\end{array}$ & $\begin{array}{c}r_{\mathrm{c}} \\
(\mathrm{fm})\end{array}$ & $\begin{array}{c}r_{\mathrm{c}}^{\text {exp. }} \\
(\mathrm{fm})\end{array}$ & Elem. $N$ & $\begin{array}{c}B \\
(\mathrm{MeV})\end{array}$ & $\begin{array}{c}B^{\text {exp. }} \\
(\mathrm{MeV})\end{array}$ & $\begin{array}{c}r_{\mathrm{c}} \\
(\mathrm{fm})\end{array}$ & $\begin{array}{c}r_{\mathrm{c}}^{\text {exp. }} \\
(\mathrm{fm})\end{array}$ & $\begin{array}{c}\text { Elem. } \\
\end{array}$ & $\begin{array}{c}B^{\text {exp. }} \\
(\mathrm{MeV})\end{array}$ & $\begin{array}{c}B^{\text {exp. }} \\
(\mathrm{MeV})\end{array}$ & $\begin{array}{c}r_{\mathrm{c}} \\
(\mathrm{fm})\end{array}$ & $\begin{array}{c}r_{\mathrm{c}} \\
(\mathrm{fm})\end{array}$ \\
\hline
\end{tabular}

\begin{tabular}{|c|c|c|c|c|c|c|c|c|c|c|c|c|c|c|c|c|c|}
\hline \multirow[t]{4}{*}{$\mathrm{Ne}$} & 6 & 99.266 & 97.321 & 3.270 & - & & 50 & 783.457 & 783.892 & 4.253 & 4.269 & Dy & 82 & 1209.677 & 1210.780 & 5.011 & 5.054 \\
\hline & 8 & 136.762 & 132.143 & 3.004 & 2.972 & & 52 & 798.416 & 799.721 & 4.275 & 4.306 & $\mathrm{Er}$ & 82 & 1213.553 & 1215.331 & 5.046 & 5.039 \\
\hline & 16 & 201.409 & 201.601 & 2.934 & 2.927 & Mo & 44 & 722.829 & 725.831 & 4.282 & - & $\mathrm{Yb}$ & 82 & 1215.892 & 1218.382 & 5.079 & 5.030 \\
\hline & 18 & 210.055 & 206.929 & 2.960 & 2.963 & & 46 & 748.226 & 750.117 & 4.289 & - & $\mathrm{Pt}$ & 92 & 1326.638 & 1327.406 & 5.285 & - \\
\hline \multirow[t]{2}{*}{$\mathrm{Mg}$} & 8 & 139.397 & 134.468 & 3.262 & - & & 50 & 795.461 & 796.508 & 4.302 & 4.316 & $\mathrm{Hg}$ & 92 & 1327.960 & 1326.766 & 5.310 & - \\
\hline & 20 & 252.470 & 249.849 & 3.095 & - & & 52 & 812.048 & 814.255 & 4.325 & 4.352 & & 94 & 1349.499 & 1348.469 & 5.322 & - \\
\hline $\mathrm{Si}$ & 20 & 284.656 & 283.429 & 3.160 & - & $\mathrm{Ru}$ & 50 & 805.152 & 806.848 & 4.348 & - & & 96 & 1370.090 & 1369.743 & 5.334 & - \\
\hline$S$ & 20 & 308.727 & 308.714 & 3.272 & 3.298 & $\mathrm{Pd}$ & 50 & 812.826 & 815.088 & 4.392 & - & & 126 & 1617.160 & 1621.049 & 5.494 & 5.485 \\
\hline \multirow[t]{2}{*}{$\mathrm{Ar}$} & 20 & 329.008 & 327.342 & 3.357 & 3.402 & & 52 & 832.905 & 836.301 & 4.414 & - & $\mathrm{Pb}$ & 96 & 1371.354 & 1368.974 & 5.354 & - \\
\hline & 22 & 345.907 & 343.810 & 3.372 & 3.427 & $\mathrm{Cd}$ & 50 & 818.559 & 821.067 & 4.432 & - & & 98 & 1392.218 & 1390.624 & 5.365 & - \\
\hline \multirow[t]{5}{*}{$\mathrm{Ca}$} & 16 & 281.114 & 281.360 & 3.426 & - & & 52 & 840.493 & 843.829 & 4.453 & - & & 100 & 1412.482 & 1411.654 & 5.376 & - \\
\hline & 18 & 314.695 & 313.122 & 3.423 & - & $\mathrm{Sn}$ & 50 & 822.373 & 824.794 & 4.468 & - & & 102 & 1432.207 & 1432.015 & 5.386 & - \\
\hline & 20 & 345.755 & 342.052 & 3.425 & 3.476 & & 52 & 846.232 & 849.086 & 4.490 & - & & 104 & 1451.439 & 1451.794 & 5.398 & - \\
\hline & 30 & 428.933 & 427.490 & 3.501 & 3.514 & & 62 & 953.239 & 953.531 & 4.576 & 4.594 & & 114 & 1541.017 & 1543.186 & 5.452 & 5.442 \\
\hline & 32 & 438.705 & 436.571 & 3.525 & - & & 64 & 971.484 & 971.574 & 4.590 & 4.610 & & 116 & 1557.666 & 1560.019 & 5.463 & 5.450 \\
\hline \multirow[t]{7}{*}{$\mathrm{Ti}$} & 18 & 316.303 & 314.491 & 3.572 & - & & 66 & 988.808 & 988.684 & 4.604 & 4.627 & & 118 & 1573.883 & 1576.354 & 5.473 & 5.459 \\
\hline & 20 & 350.990 & 346.905 & 3.540 & - & & 68 & 1005.287 & 1004.954 & 4.618 & 4.641 & & 120 & 1589.641 & 1592.187 & 5.483 & 5.469 \\
\hline & 22 & 374.927 & 375.475 & 3.535 & - & & 70 & 1021.023 & 1020.546 & 4.633 & 4.6 & & 122 & 1604.897 & 1607.506 & 5.492 & 5.479 \\
\hline & 26 & 418.185 & 418.699 & 3.549 & 3.591 & & 72 & 1036.100 & 1035.529 & 4.647 & 4.666 & & 124 & 1619.568 & 1622.324 & 5.501 & 5.490 \\
\hline & 28 & 436.198 & 437.781 & 3.559 & 3.570 & & 74 & 1050.567 & 1049.963 & 4.661 & 4.676 & & 126 & 1633.472 & 1636.430 & 5.509 & 5.501 \\
\hline & 30 & 450.512 & 451.962 & 3.582 & - & & 76 & 1064.429 & 1063.889 & 4.676 & - & & 128 & 1642.984 & .552 & 5.529 & 5.523 \\
\hline & 32 & 463.004 & 464.234 & 3.606 & - & & 78 & 1077.635 & 1077.346 & 4.690 & - & & 130 & 1652.223 & 1654.514 & 5.548 & 5.545 \\
\hline \multirow[t]{2}{*}{$\mathrm{Cr}$} & 22 & 380.810 & 381.978 & 3.625 & - & & 80 & 1090.071 & 1090.293 & 4.703 & - & & 132 & 1661.295 & 1663.291 & 5.565 & 5.565 \\
\hline & 28 & 452.793 & 456.349 & 3.627 & 3.642 & & 82 & 1101.452 & 1102.851 & 4.715 & - & Po & 120 & 1597.316 & 1599.165 & 5.517 & 5.503 \\
\hline \multirow{4}{*}{$\mathrm{Ni}$} & 38 & 577.084 & 576.808 & 3.848 & - & $\mathrm{Xe}$ & 80 & 1127.656 & 1127.434 & 4.789 & 4.792 & & 124 & 1637.270 & 1637.293 & 5.567 & 5.544 \\
\hline & 40 & 592.287 & 590.408 & 3.866 & - & & 82 & 1142.487 & 1141.877 & 4.799 & 4.799 & & 126 & 1652.787 & 1652.497 & 5.574 & 5.554 \\
\hline & 42 & 604.799 & 602.236 & 3.886 & - & & 84 & 1151.416 & 1151.746 & 4.822 & 4.836 & & 128 & 1664.614 & 1664.300 & 5.594 & - \\
\hline & 44 & 615.703 & 613.169 & 3.906 & - & $\mathrm{Ba}$ & 80 & 1144.124 & 1142.775 & 4.828 & 4.833 & & 130 & 1676.101 & 1675.867 & 5.613 & - \\
\hline \multirow[t]{3}{*}{$\mathrm{Zn}$} & 28 & 480.525 & 486.964 & 3.840 & - & & 82 & 1160.594 & 1158.292 & 4.838 & 4.838 & $\mathrm{Ra}$ & 122 & 1627.084 & 1625.669 & 5.590 & 5.554 \\
\hline & 40 & 610.734 & 611.086 & 3.938 & 3.985 & & 84 & 1170.762 & 1169.444 & 4.862 & 4.870 & & 124 & 1644.283 & 1642.464 & 5.598 & 5.562 \\
\hline & 42 & 625.597 & 625.796 & 3.955 & - & $\mathrm{Ce}$ & 80 & 1158.826 & 1156.034 & 4.865 & 4.873 & & 126 & 1660.562 & 1658.315 & 5.605 & 5.571 \\
\hline $\mathrm{Ge}$ & 50 & 700.989 & 702.437 & 4.080 & - & & 82 & 1176.896 & 1172.692 & 4.875 & 4.877 & & 128 & 1673.586 & 1671.267 & 5.625 & - \\
\hline \multirow[t]{2}{*}{$\mathrm{Se}$} & 50 & 727.463 & 727.343 & 4.126 & - & & 84 & 1188.434 & 1185.289 & 4.899 & 4.907 & & 130 & 1686.209 & 1684.050 & 5.644 & - \\
\hline & 52 & 737.305 & 738.074 & 4.153 & - & & 86 & 1199.632 & 1197.330 & 4.921 & 4.931 & $\mathrm{Th}$ & 122 & 1632.040 & 1628.617 & 5.621 & - \\
\hline \multirow[t]{2}{*}{$\mathrm{Kr}$} & 50 & 749.164 & 749.234 & 4.169 & 4.184 & $\mathrm{Nd}$ & 80 & 1168.468 & 1167.295 & 4.901 & 4.910 & & 124 & 1650.041 & 1646.139 & 5.628 & - \\
\hline & 52 & 760.633 & 761.804 & 4.195 & 4.217 & & 82 & 1187.830 & 1185.141 & 4.909 & 4.912 & & 126 & 1667.060 & 1662.689 & 5.634 & - \\
\hline \multirow[t]{3}{*}{$\mathrm{Sr}$} & 48 & 749.276 & 748.928 & 4.201 & 4.226 & & 84 & 1200.491 & 1199.082 & 4.932 & 4.941 & & 128 & 1681.298 & 1676.762 & 5.655 & - \\
\hline & 50 & 767.989 & 768.468 & 4.209 & 4.220 & $\mathrm{Sm}$ & 80 & 1176.227 & 1176.614 & 4.935 & 4.944 & & 130 & 1695.061 & 1690.610 & 5.673 & - \\
\hline & 52 & 781.200 & 782.631 & 4.233 & 4.261 & & 82 & 1196.890 & 1195.736 & 4.942 & 4.944 & & 126 & 1672.236 & 1665.648 & 5.664 & - \\
\hline $\mathrm{Zr}$ & 46 & 740.699 & 740.644 & 4.237 & - & & 84 & 1210.720 & 1210.909 & 4.965 & 4.975 & $\mathrm{U}$ & 132 & 1717.151 & 1710.285 & 5.719 & - \\
\hline & 48 & 762.784 & 762.605 & 4.246 & 4.281 & $\mathrm{Gd}$ & 82 & 1204.158 & 1204.435 & 4.977 & 4.976 & & & & & & \\
\hline
\end{tabular}


TABLE IV. Specifications of the $\chi^{2}$ definition. The total $\chi^{2}$ found for DD-ME $\delta$ is almost 40; the partial contributions to it are listed in the fifth column.

\begin{tabular}{lcrccc}
\hline \hline $\mathcal{O}_{i}$ & $w_{i}$ & $n_{\text {data }}$ & $\rho\left(\mathrm{fm}^{-3}\right)$ & $\chi^{2}$ & Ref. \\
\hline$B$ & $1 / 0.50 \mathrm{MeV}^{-1}$ & 161 & & 23.40 & {$[80]$} \\
$r_{\mathrm{c}}$ & $1 / 0.01 \mathrm{fm}^{-1}$ & 86 & & 2.90 & {$[82]$} \\
$e(\rho, \alpha=1)$ & $1 /\left(0.03 \times \mathcal{O}_{i}\right)$ & 30 & $0.01-0.30$ & 3.42 & {$[19]$} \\
$e(\rho, \alpha=0)$ & $1 /\left(0.03 \times \mathcal{O}_{i}\right)$ & 30 & $0.01-0.30$ & 7.03 & {$[19]$} \\
$m_{p}^{*}-m_{n}^{*}$ & $1 /\left(0.03 \times \mathcal{O}_{i}\right)$ & 25 & $0.04-0.20$ & 0.39 & {$[65]$} \\
\hline \hline
\end{tabular}

the constant-gap approximation with gap parameters derived from the odd-even mass differences. For a full description of nuclei all over the periodic table, which includes also regions where the experimental binding energies are not known, we introduce a more general description of pairing by means of a monopole force with a constant matrix element fitted to reproduce the experimental binding energies of the nuclei in Table III. We obtain for the set DD-ME $\delta$ the values $G_{n}=32.44 / A \mathrm{MeV}$ for neutrons and $G_{p}=29.76 / A \mathrm{MeV}$ for protons. In order to have a fair comparison for the results in finite nuclei we treated in the following the pairing properties of the set DD-ME2 also by a monopole force. In a similar fit we found for DD-ME2 the strength parameters $G_{n}=29.86 / A \mathrm{MeV}$ and $G_{p}=28.92 / A \mathrm{MeV}$. In all these calculations the soft pairing window described in Ref. [78] has been used.

\section{RESULTS}

\section{A. Nuclear and neutron matter equations of state}

The nuclear matter properties at saturation computed with the DD-ME $\delta$ functional are given in Table V. These properties do not fully coincide with the ones of the fully microscopic calculation in [19]. The reason for that is that, in the microscopic calculation, the EoS is very flat around saturation density and some deviation between the microscopic results and the DD-ME $\delta$ fit appear. These differences remain within the uncertainty of the state of the art of present numerical microscopic calculations. They are too small to be seen on the scale of Fig. 1. They are, however, important for a fine tuning of the results.

In order to investigate the quality of the predictions of the density functional DD-ME $\delta$ in the high-density domain, we show in Fig. 3 the pressure (27) computed with this functional as a function of the density. It is compared with the pressure

TABLE V. Nuclear saturation properties as predicted by the parameter sets DD-ME $\delta$ and DD-ME2.

\begin{tabular}{lccc}
\hline \hline & DD-ME $\delta$ & DD-ME2 & \\
\hline$\rho_{\text {sat }}$ & 0.152 & 0.152 & $\left(\mathrm{fm}^{-3}\right)$ \\
$e_{\text {sat }}$ & -16.12 & -16.14 & $(\mathrm{MeV})$ \\
$K_{\infty}$ & 219.1 & 250.89 & $(\mathrm{MeV})$ \\
$J$ & 32.35 & 32.30 & $(\mathrm{MeV})$ \\
$L$ & 52.85 & 51.26 & $(\mathrm{MeV})$ \\
$m^{*} / m$ & 0.609 & 0.572 & \\
\hline \hline
\end{tabular}

derived from the microscopic calculation of Ref. [19] as well as with the results derived from the nonlinear meson coupling models NL3 [4] and FSUGold [9] and from DD-ME2 [10]. We see that both microscopic and DD-ME $\delta$ calculations are within the shaded area which corresponds to the "experimental region" estimated from simulations of heavy-ion collisions [83]. The standard nonlinear $\sigma-\omega$ model NL3 is outside of this region while the FSUGold model—which has an additional nonlinear $\omega$ - $\rho$ coupling that softens the symmetry energy (see below) - is inside the shaded area, in rather good agreement with DD-ME $\delta$ and the microscopic results. The results of the parameter set DD-ME2 are slightly outside of the shaded area.

An important quantity in nuclear physics and astrophysics, directly related to the EoS of asymmetric nuclear matter, is the symmetry energy (30). The value of the symmetry energy derived from successful mean-field models lies roughly in a window of $30-35 \mathrm{MeV}$ at saturation. However, the density dependence of the symmetry energy is much more uncertain. This fact entails important consequences for a number of isospin-dependent observables. As a paradigmatic example, one may recall that different accurate mean-field models which reproduce well the binding energy and charge radius of the nucleus ${ }^{208} \mathrm{~Pb}$ predict largely different values for the neutron skin thickness of this isotope, ranging from 0.1 to $0.3 \mathrm{fm}$. This fact points out that the isovector properties of the different models are, actually, not well constrained by the binding energies and charge radii of stable finite nuclei used to fit the effective interactions.

In nuclear mean-field models, a strong linear correlation exists $[84,85]$ between the size of the neutron skin thickness of a heavy neutron-rich nucleus such as ${ }^{208} \mathrm{~Pb}$ and the $L$ parameter defined in Eq. (39), i.e., the slope of the symmetry energy at saturation. Recent constraints on the $L$ parameter have been obtained using a variety of observables such as, for instance, isospin diffusion [86-88] and isoscaling [89-93] in heavy-ion reactions, some collective excitations in nuclei [71,94-96], and the neutron skin thickness in finite nuclei $[97,98]$ measured in antiprotonic atoms $[99,100]$. The analysis of all these results suggests that the $L$ parameter is roughly within the window 45-75 MeV [97]. The new experimental efforts to measure the neutron radius of ${ }^{208} \mathrm{~Pb}$ may turn out in the future to be helpful for deducing narrower constraints on the slope $L$ of the symmetry energy through the correlation of $L$ with the neutron skin thickness [85,101].

The $L$ value predicted by our DD-ME $\delta$ functional is $53 \mathrm{MeV}$. It is close to the result of the microscopic calculation of $56 \mathrm{MeV}$ in Ref. [12] and of $66.5 \mathrm{MeV}$ in Ref. [104]. The density dependence of the symmetry energy exhibited by $\mathrm{DD}-\mathrm{ME} \delta$ is displayed in Fig. 4 . We see that DD-ME $\delta$ predicts a rather soft density dependence of the symmetry energy, which lies inside the shaded region derived from the empirical law $S_{2}(\rho)=31.6\left(\rho / \rho_{0}\right)^{\gamma} \mathrm{MeV}$, imposing the range discussed above: $45<L<75 \mathrm{MeV}$. It turns out that the density dependence of DD-ME $\delta$ and DD-ME2 is practically the same. This fact is not trivial, first, because DD-ME2 has not been adjusted to nuclear matter data, but only to the experimental skin thickness of several finite nuclei [10], and, second, because the full isospin dependence is determined by the $\rho$ meson, whereas in DD-ME $\delta$ it is distributed over the $\delta$ 


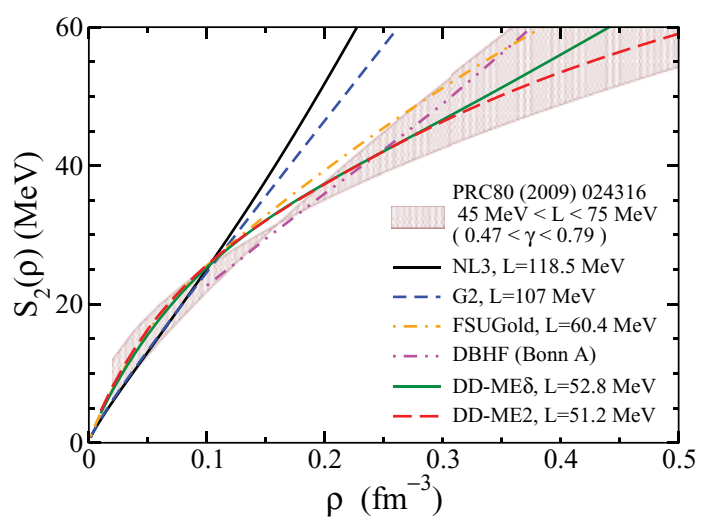

FIG. 4. (Color online) The symmetry energy in Eq. (29) as a function of the density. The results for DD-ME $\delta$ are compared with those of the density functionals NL3 from Ref. [4], G2 from Ref. [102], FSUGold from Ref. [9], and microscopic DBHF calculations from Ref. [103]. The shaded area represents the empirical region suggested by the available constraints on the $L$ parameter discussed in [97].

and the $\rho$ meson. The reason for this good agreement can be understood from the upper panel of Fig. 5 where the different contributions to the symmetry energy are displayed, the kinetic

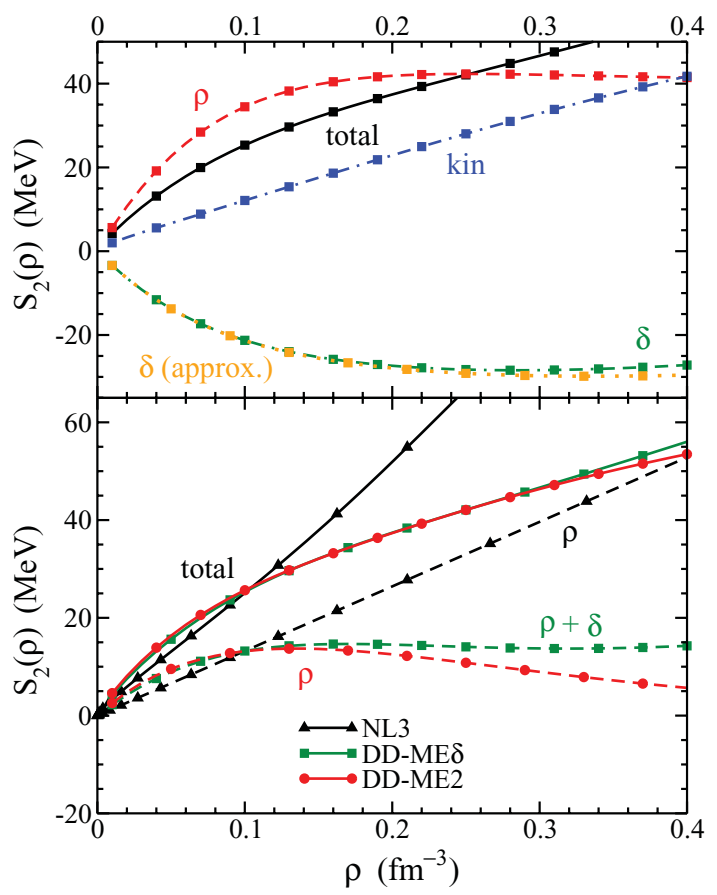

FIG. 5. (Color online) Upper panel: The symmetry energy $S_{2}(\rho)$ (full in black) and its contributions as a function of the density, the kinetic contribution (dash dotted in blue), the contributions of the $\rho$ meson (dashed in red) and the $\delta$ meson (dash dotted in green), and approximation (37) for the contribution of the $\delta$ meson (dotted in yellow) for DD-ME $\delta$. Lower panel: The symmetry energy $S_{2}(\rho)$ resulting from the parameter sets NL3 (triangles), DD-ME2 (circles), and DD-ME $\delta$ (squares). The total value (solid line) is compared with the contribution of the $\rho$ meson (dashed line) for NL3 and DD-ME2 and of the sum of $\rho$ and $\delta$ mesons for DD-ME $\delta$.

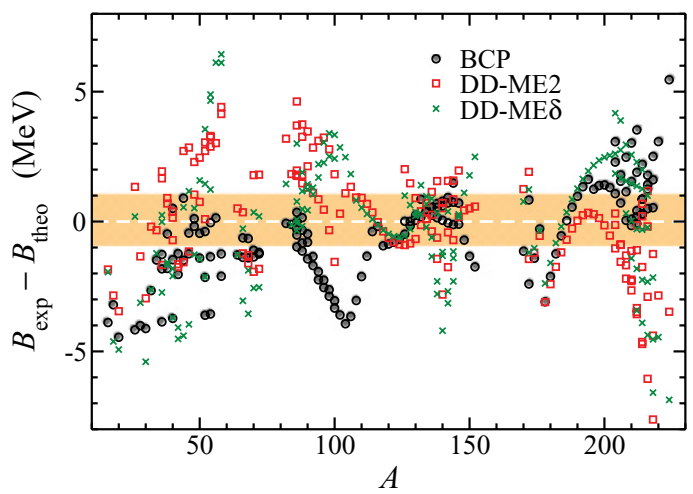

FIG. 6. (Color online) Difference between theoretical and experimental binding energies as a function of the mass number. Results of the functional DD-ME $\delta$ are compared with those of DD-ME2 [10] and of BCP [12]. The orange region corresponds to twice the fixed weight used in the fit (see Table IV).

part as well as those provided by the $\rho$ and the $\delta$ meson. We can see that the contributions of these mesons have opposite sign and thus a noticeable cancellation appears between them over the entire range of densities under consideration. Thus, it is conceivable [see Eq. (40)] that if the $\delta$ meson is not considered in the functional (as is the case of DD-ME2) its contribution to the symmetry energy can be accounted for by the $\rho$ meson (with a reduced strength of the coupling constant; see Ref. [10] and Table II).

The lower panel shows similar decompositions of the symmetry energy for other density functionals, such as NL3 [4] and DD-ME2 [10]. The parameter set NL3 (black line) has no density dependence in the isovector channel. Therefore the contribution of the $\rho$ meson is very stiff and proportional to the density. The parameter set DD-ME2 (red line) includes only one isovector meson, the $\rho$ meson, and its contribution to the symmetry energy is very close the the sum of both the $\rho$ and the $\delta$ meson for the set DD-ME $\delta$ (green line), which compensate each other to a large extent. Small differences in these curves at densities above saturation density can be traced

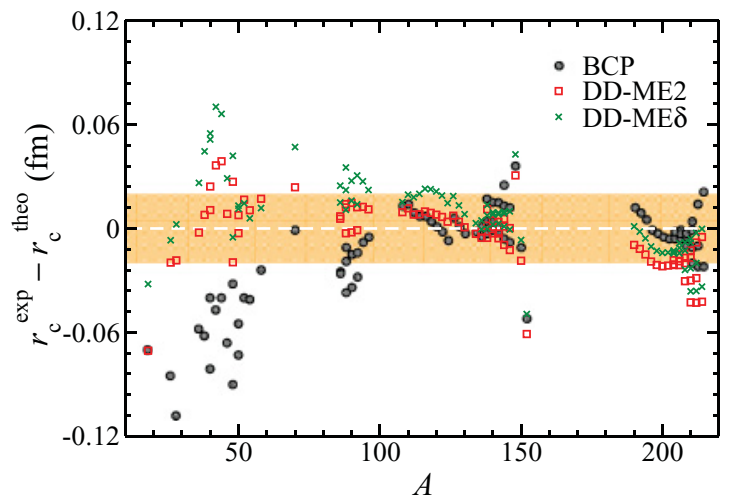

FIG. 7. (Color online) Difference between theoretical and experimental charge radii as a function of the mass number. DD-ME $\delta$ results are compared with those of DD-ME2 [10] and of BCP [12]. The orange region corresponds to twice the fixed weight used in the fit (see Table IV). 


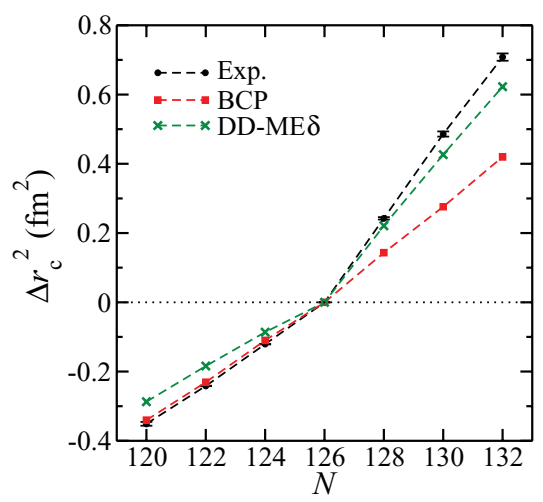

FIG. 8. (Color online) Isotope shifts for the chain of $\mathrm{Pb}$ isotopes with respect to ${ }^{208} \mathrm{~Pb}$. Calculations with the relativistic model DD$\mathrm{ME} \delta$ and the nonrelativistic functional $\mathrm{BCP}$ are compared.

back to the different ansatze for the density dependence of the $\rho$ meson in these two parameter sets, the Typel-Wolter ansatz (46) for DD-ME $\delta$ and an exponential density dependence for DD-ME2 (see Eq. (7) in Ref. [10]).

\section{B. Ground-state properties of finite nuclei}

As described in Sec. III A the experimental masses of 161 and the charge rms radii of 86 even-even spherical nuclei (see Table III) have been taken into account in the fitting procedure of the DD-ME $\delta$ functional.

We display in Figs. 6 and 7 the difference between theoretical results computed with the functionals DD-ME $\delta$, DD-ME2, and BCP and experimental data. For DD-ME $\delta$ we obtain a rms deviations of $2.4 \mathrm{MeV}$ for the binding energies and $0.02 \mathrm{fm}$ for the charge radii. These results are close to the rms deviations of $2.1 \mathrm{MeV}$ and $0.02 \mathrm{fm}$ obtained with the DD-ME2 functional for the same set of data when pairing correlations are introduced by the monopole force discussed at the end of Sec. III B. It has to be emphasized, however, that using the density functional DD-ME2 in connection with the pairing part of the finite-range Gogny force D1S instead of the

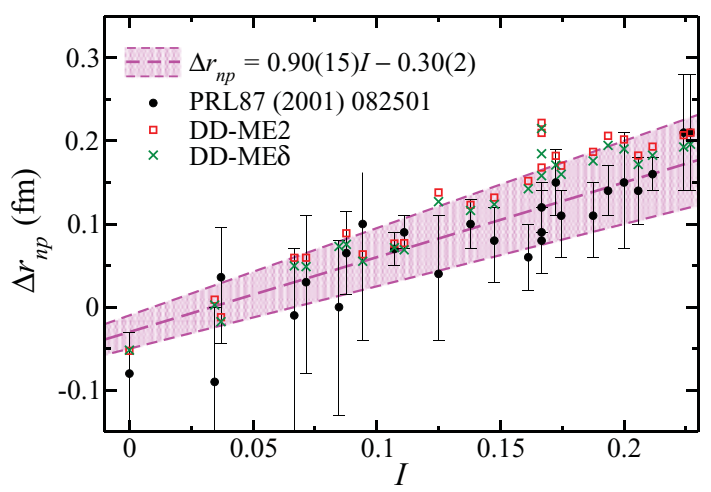

FIG. 9. (Color online) The neutron skin thickness $\Delta r_{n p}=$ $\left\langle r^{2}\right\rangle_{n}^{1 / 2}-\left\langle r^{2}\right\rangle_{p}^{1 / 2}$ as a function of the asymmetry parameter $I=$ $(N-Z) /(N+Z)$. Results obtained with the parameter set DD-ME $\delta$ are compared with those of the set DD-ME2 [10] and experimental values [99]. monopole force and taking into account spherical as well as deformed nuclei one has found rms deviations of $900 \mathrm{keV}$ and $0.017 \mathrm{fm}$ for the binding energies and charge radii of typical sets of 200 [10] or 300 [105] even-even nuclei.

The charge radii $r_{c}$ [defined in Eq. (48)] of $\mathrm{Pb}$ isotopes and their isotope shifts have been a matter of detailed discussion within the framework of mean-field theories [106-109]. In Fig. 8 we show the isotope shifts in a chain of $\mathrm{Pb}$ isotopes as a function of the neutron number $N$. The nucleus ${ }^{208} \mathrm{~Pb}$ has been taken as the reference point: $\Delta_{r_{c}}^{2}(N)=r_{c}^{2}(N)-r_{c}^{2}(126)$. With a gradual addition of neutrons, the empirical charge radii of isotopes heavier than ${ }^{208} \mathrm{~Pb}$ do not show the trend of the lighter isotopes and at the doubly magic nucleus ${ }^{208} \mathrm{~Pb}$ one observes a pronounced kink [110]. Conventional nonrelativistic Skyrme and Gogny forces fail to reproduce this kink [106], whereas all the relativistic models are successful in describing this kink properly [107]. In Refs. [108,109] this difference between the nonrelativistic Skyrme functional and the relativistic models has been traced back to the isospin dependence of the spinorbit force. In conventional relativistic models it is determined by the $\rho$-meson vertex and it is relatively weak. In Fig. 8 we see that the parameter set DD-ME $\delta$ reproduces the kink in the isotope shifts rather well as all the other relativistic models do. The nonrelativistic set BCP of Ref. [12], which has the same spin-orbit force as conventional Skyrme and Gogny functionals, fails in this context.

Finally, we show in Fig. 9 values for the neutron skin thickness $\Delta r_{n p}=\left\langle r^{2}\right\rangle_{n}^{1 / 2}-\left\langle r^{2}\right\rangle_{p}^{1 / 2}$ of a large set of nuclei as a function of the relative neutron excess $I=(N-Z) /(N+Z)$ and compare the results obtained with the parameter set DD-ME $\delta$ with those of the set DD-ME2 and with experimental values [99]. Both theoretical calculations are in rather good agreement and within the range of the experimental error bars.

\section{Impact of the $\delta$ meson on the spin-orbit splitting}

In this work, we have included the $\delta$ meson in our theoretical treatment of the nucleus motivated by microscopic calculations $[42,43,65]$ and by the importance of a scalar-isovector meson of the nucleon-nucleon potentials for describing the nucleonnucleon scattering data in vacuum [72]. In our investigation of the properties of nuclear matter we have seen in Fig. 5 that the influence of the $\delta$ meson on the symmetry energy can be largely compensated by renormalizing the $\rho$-meson coupling constant in the DD-ME2 model. The same seems to be true also for the masses (Fig. 6), radii (Fig. 7), and skin thicknesses (Fig. 9) in finite nuclei. Obviously this also applies for all the other successful covariant density functionals without the $\delta$-meson degree of freedom.

In order to get a better understanding of these results we follow Ref. [111] and eliminate the small components of the spinor $\psi_{i}$ in the Dirac equation (5). For the large components $f_{i}(\mathbf{r})$ we are left with a Schrödinger-like equation

$$
\left\{\sigma p \frac{1}{2 m+\varepsilon_{i}+V_{-}} \sigma p+V_{+}\right\} f_{i}=\varepsilon_{i} f_{i} .
$$

It contains the potentials

$$
V_{ \pm}=\Sigma^{s} \pm \Sigma^{0} .
$$


The potential $V_{+} \approx-50 \mathrm{MeV}$ corresponds to the conventional potential in the corresponding nonrelativistic Schrödinger equation. In theories containing $\rho$ and the $\delta$ mesons it can be decomposed into an isoscalar and an isovector part:

$$
V_{+}(r)=V_{+}^{\mathrm{IS}}(r)+\tau_{3} V_{+}^{\mathrm{IV}}(r),
$$

with

$$
\begin{aligned}
V_{+}^{\mathrm{IS}}(r) & =g_{\sigma} \sigma(r)+g_{\omega} \omega^{0}(r), \\
V_{+}^{\mathrm{IV}}(r) & =g_{\delta} \delta_{3}(r)+g_{\rho} \rho_{3}^{0}(r),
\end{aligned}
$$

where $\sigma(r), \omega^{0}(r), \delta_{3}(r)$, and $\rho_{3}^{0}(r)$ are the corresponding meson fields. In theories without the $\delta$ meson the $\rho$-meson vertex has to be renormalized and we find for the isovector part a pure $\rho$ field,

$$
V_{+}^{\mathrm{IV}}(r)=\tilde{g}_{\rho} \rho_{3}^{0}(r),
$$

with a renormalized coupling $\tilde{g}_{\rho}$. Since the fields $\delta_{3}(r)$ and $\rho_{3}^{0}(r)$ have opposite sign the renormalized coupling $\tilde{g}_{\rho}$ has to be considerably smaller than the original $g_{\rho}$, as is seen in Tables I and II.

The situation is different for the potential $V_{-} \approx-700 \mathrm{MeV}$, which leads to a very large spin-orbit term. In spherical nuclei it has a strength $U_{\tau}^{\text {so }}(r)$ for neutrons and protons $(\tau=n, p)$ of the form

$$
U_{\mathrm{so}}^{\tau}(r) \equiv \frac{1}{2 m} \frac{1}{2 m+V_{-}^{\tau}} \frac{1}{r} \frac{\partial V_{-}^{\tau}}{\partial r} .
$$

Because of the nonlinear connection between $U_{\text {so }}$ and $V_{-}$the situation is more complicated here and we obtain the isoscalar and isovector parts as

$$
\begin{aligned}
& U_{\mathrm{so}}^{\mathrm{IS}}=\frac{1}{2}\left(U_{\mathrm{so}}^{n}+U_{\mathrm{so}}^{p}\right), \\
& U_{\mathrm{so}}^{\mathrm{IV}}=\frac{1}{2}\left(U_{\mathrm{so}}^{n}-U_{\mathrm{so}}^{p}\right) .
\end{aligned}
$$

Nonetheless, these terms are dominated by $V_{-}(r)$ and the decomposition of this function with respect to isospin is

$$
\begin{aligned}
V_{-}^{\mathrm{IS}}(r) & =g_{\sigma} \sigma(r)-g_{\omega} \omega^{0}(r), \\
V_{-}^{\mathrm{IV}}(r) & =g_{\delta} \delta_{3}(r)-g_{\rho} \rho_{3}^{0}(r) .
\end{aligned}
$$

Because of the opposite sign of the fields $\sigma$ and $\omega$ the corresponding isoscalar part of the spin-orbit potential is considerably enhanced with respect to the isoscalar part of the normal potential. This well-known fact is also true for the isovector part. It is also considerably enhanced with respect to the isovector part of the normal potential. As a consequence there is an essential difference between theories with and without a $\delta$ meson; i.e., we expect an enhancement of the isospin dependence of the spin-orbit potential in a theory with a $\delta$ meson.

To clarify these statements we show in Fig. 10 the isoscalar and the isovector parts of the spin-orbit potential defined in Eqs. (60) and (61) for the three Sn isotopes ${ }^{100} \mathrm{Sn},{ }^{132} \mathrm{Sn}$, and ${ }^{176} \mathrm{Sn}$. In order to have clear evidence for the isospin dependence we neglect in this case the Coulomb potential.

In the first panel $\left({ }^{100} \mathrm{Sn}\right)$ we have $N=Z$ and because the Coulomb potential has been neglected the neutron and proton densities are identical. As a consequence, the isovector meson fields $\delta(r)$ and $\rho(r)$, as well as the isovector part of
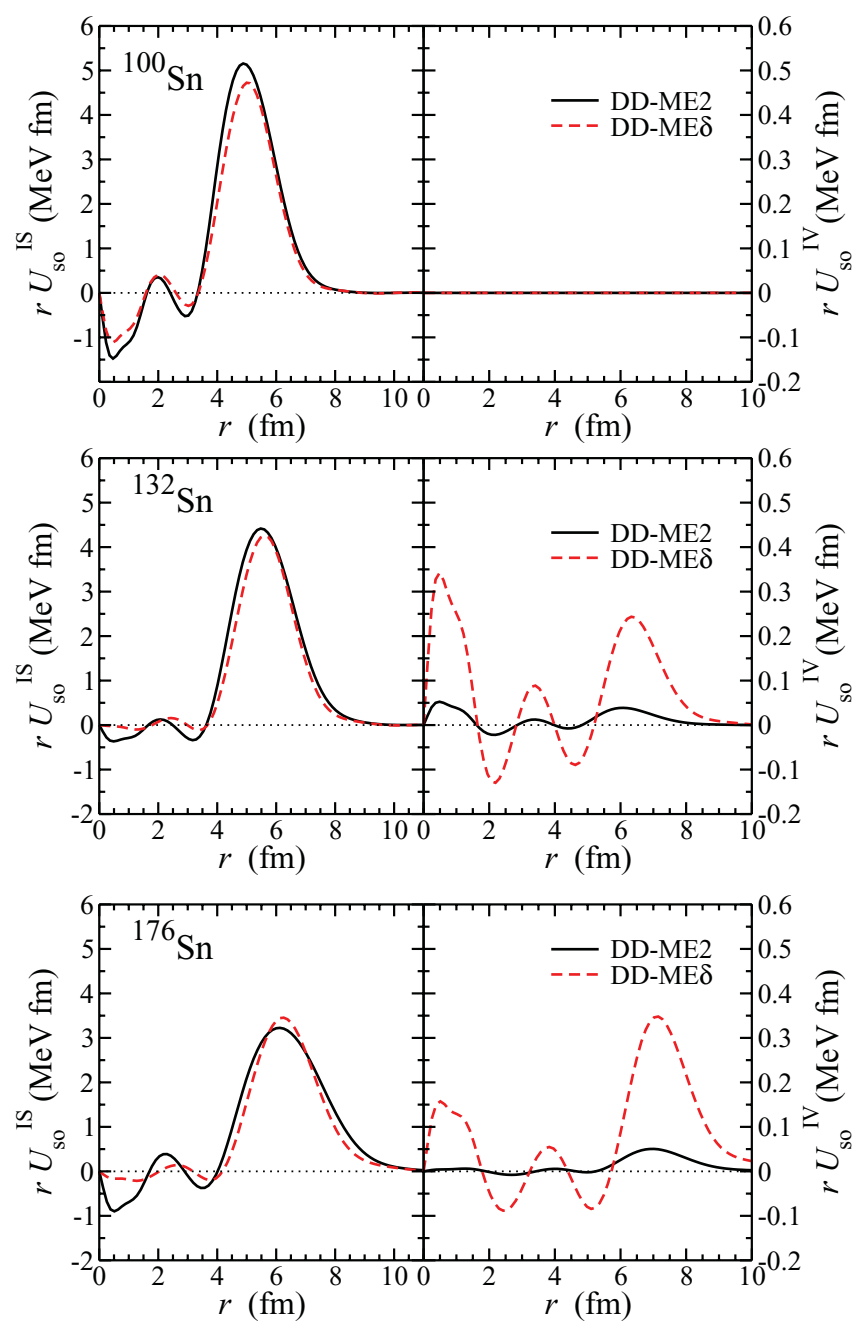

FIG. 10. (Color online) The isoscalar part (left panel) and the isovector part (right panel) of the spin-orbit potential for the $\mathrm{Sn}$ isotopes ${ }^{100} \mathrm{Sn},{ }^{132} \mathrm{Sn}$, and ${ }^{176} \mathrm{Sn}$.

the spin-orbit potential, vanish identically. This is true for both models DD-ME $\delta$ and DD-ME2. Because of the different coupling constants in these models the isoscalar parts of the spin-orbit potentials are slightly different, but they are large in both cases and peaked at the surface. In the second panel $\left({ }^{132} \mathrm{Sn}\right)$ we have a considerable neutron excess. The isoscalar part did not change very much. Apart from the fact that the larger mass number $A=132$ produces a shift of the surface and the maximum of the spin-orbit potential to larger $r$ values, both models show similar results. The situation is very different for the isovector part. For the DD-ME2 model without the $\delta$ meson the $\rho$ field is not vanishing but relatively small compared to the isoscalar fields. The effective coupling for the $\rho$-exchange value of $g_{\rho}^{2}$ is considerably smaller than the other two couplings for the other two mesons, $\sigma$ or $\omega$. In addition the source of the $\rho$ field is the difference between the neutron and the proton density. This difference is even for $N=82$ and not extremely large. However, for the DD-ME $\delta$ model with a $\delta$ and a $\rho$ meson the isovector part of the spin-orbit field is considerably enhanced with respect to the isovector part of the DD-ME2 model. However, since this is relatively small, 


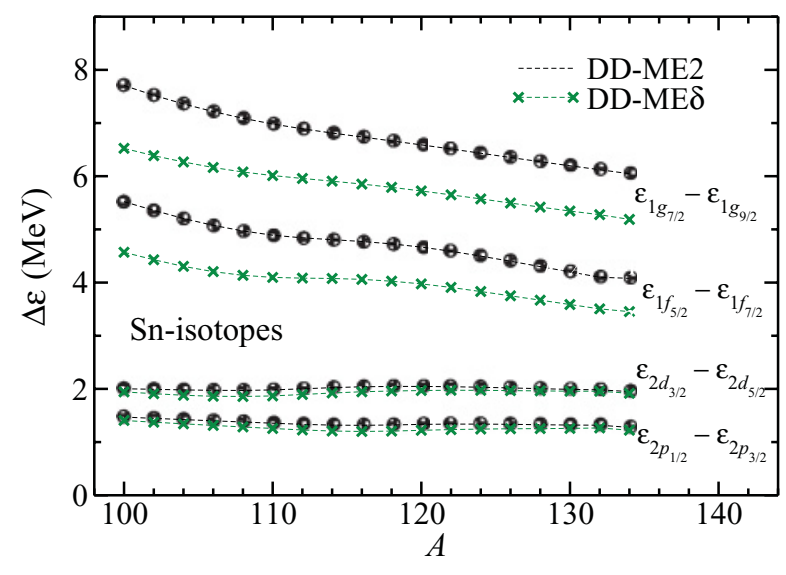

FIG. 11. (Color online) Spin-orbit splitting in Sn isotopes for various $n l$ levels. Results for the parameter set DD-ME $\delta$ (green crosses) are compared with those of the parameter set DD-ME2 (black circles).

in total the isovector part of the spin-orbit potential is still an order of magnitude smaller than the isoscalar part. Therefore even for effects that depend on the spin-orbit potential we do not expect essential differences between models with and without a $\delta$ meson. This is even true for cases with extreme neutron excess, as in the nucleus ${ }^{176} \mathrm{Sn}$ in the lowest panel of Fig. 10.

In Fig. 11 we show the spin-orbit splitting for neutron orbitals in a chain of Sn isotopes, starting at the $N=Z$ nucleus ${ }^{100} \mathrm{Sn}$. In contrast to Fig. 10 the Coulomb interaction is included here. Results for the two parameter sets DD-ME2 (without a $\delta$ meson) and DD-ME $\delta$ (with a $\delta$ meson) are compared. First, we find that the difference of these two models is rather small for the $2 p$ and the $2 d$ orbits. Because of the low $\ell$ values these splittings are relatively small and the corresponding wave functions are not so surface peaked. For the $1 f$ and the $1 g$ orbits with large $\ell$ values the splitting is relatively large and we find a considerable difference between the DD-ME2 and the DD-ME $\delta$ models. This difference is, however, connected with the isoscalar part of the spin-orbit potential, because it occurs already in the nucleus ${ }^{100} \mathrm{Sn}$, which has, apart from a small violation of isospin due to the Coulomb force, practically no isovector part.

With increasing neutron number the spin-orbit splitting in these high- $\ell$ orbitals decreases. This has already been observed in earlier investigations in Refs. [112] and [113], where it has been explained by the increasing neutron skin and the increasing neutron diffuseness leading to a reduced derivative in the spin-orbit potential.

Finally, we observe in Fig. 11 that the difference in the neutron spin-orbit splittings calculated with DD-ME2 and DDME $\delta$ decreases slightly with increasing neutron number. This effect has its origin in the increasing isovector part of the neutron spin-orbit splitting for the parameter set DD-ME $\delta$ : $U_{\mathrm{so}}^{n}=\left(U_{\mathrm{so}}^{\mathrm{IS}}+U_{\mathrm{so}}^{\mathrm{IV}}\right) / 2$. However, it is relatively small because the isovector part itself is small compared to the isoscalar part (see the scales on the right panels in Fig. 10). Of course, for the protons with $U_{\mathrm{so}}^{p}=\left(U_{\mathrm{so}}^{\mathrm{IS}}-U_{\mathrm{so}}^{\mathrm{IV}}\right) / 2$ this difference increases with increasing neutron number (not shown in Fig. 11).

\section{DISCUSSION AND CONCLUSIONS}

On the way to a more microscopic derivation of relativistic nuclear energy density functionals, we started with Brueckner calculations [19] for symmetric nuclear matter and pure neutron matter and with Dirac Brueckner calculations [65] for pure neutron matter. We tried to use this microscopic information as much as possible for the adjustment of a new covariant density functional based on density-dependent meson exchange. Since it is well known that, at present, all attempts to derive the functionals directly from bare forces do not reach the required accuracy for nuclear structure applications we added experimental data in finite nuclei, such as binding energies and charge radii, for the fit. In contrast to Ref. [16], where a similar idea has been applied to a relativistic point coupling model, we took into account in this work the fact that in Dirac Brueckner calculations the resulting scalar self-energies show a strong isovector part by including a $\delta$ meson, which is usually neglected in relativistic meson exchange models.

This investigation is in some sense an extension of earlier nonrelativistic work in Refs. [12]. We want to point out, however, that in the present work we were forced to leave, in part, the strategy of Ref. [12]. In that paper and in earlier work of Fayans [114] the strict Kohn-Sham strategy was followed as in Coulombic systems. Namely, the bulk part was exclusively determined from previous microscopic Brueckner calculations [19] and thus fixed once and for all. Then a phenomenological finite-range contribution and a spin-orbit term was added to the functional to account for properties of finite nuclei in adjusting four parameters. Since in the relativistic case the spin-orbit is fixed already from the nuclear matter calculations [37], if all the parameters of the relativistic approach which survive in the infinite matter limit were adjusted to microscopic infinite matter results, one would essentially remain with only one adjustable parameter, i.e., the $\sigma$ mass, which can serve for the adjustment to properties of finite nuclei. The $\omega$ and the $\rho$ mass cannot be fixed independently from present data and therefore they are kept at their experimental values in vacuum. It turns out, however, that only one parameter is not enough to reach the required accuracy for nuclear masses and radii. We, therefore, had to adjust parameters simultaneously to microscopic bulk properties and experimental finite-nuclei data, thus departing from the strict Kohn-Sham strategy. Nevertheless, we ensured that in our model the density dependence of the meson exchange couplings is completely determined by the microscopic calculations of infinite matter and only four remaining parameters are adjusted to experimental data in finite nuclei.

We have to keep in mind that the nuclear many-body problem is much more complicated than Coulombic systems. It is based on QCD, a relativistic theory, where spin degrees of freedom play an essential role, not only in the spin-orbit term. Relativistic models provide a consistent treatment of the spin degrees of freedom and velocity-dependent terms; they include the complicated interplay between the large Lorentz scalar and vector self-energies induced at the QCD level by the in-medium changes of the scalar and vector quark condensates [115]. In particular, they include nuclear magnetism, i.e., the nuclear currents induced by the spatial 
parts of the vector self-energies or the time-odd components of the nuclear density density functional. Using the Kohn-Sham strategy applied in Ref. [12] one has, at present, no possibility of deriving this part of the functional.

As a result of our investigations we have derived a functional DD-ME $\delta$ with properties similar to the very successful functional DD-ME2. In contrast to that model, DD-ME $\delta$ is based to a large extent on microscopic calculations. Only four parameters had to be adjusted to finite nuclei. It turns out that the inclusion of the $\delta$ meson does not improve the accuracy of the properties of finite nuclei such as masses and radii. Therefore the corresponding vertex and its density dependence are completely determined by nuclear matter data such as the isovector part of the effective Dirac mass. It is nevertheless much more physical, and notably the mass splitting of neutrons and protons is now correctly incorporated. It has, moreover, an influence on the behavior of the equation of state at higher densities and we find in this region a much better agrement with experimental data derived from heavy-ion reactions [83] than the earlier parameter set DD-ME2 not including the $\delta$ meson.
We therefore can hope that the parameter set derived in this investigation is more reliable for applications of relativistic density functional theory to neutron stars.

\section{ACKNOWLEDGMENTS}

We thank M. Baldo, G. A. Lalazissis, L. M. Robledo, B. K. Sharma, and D. Vretenar for helpful discussions and C. Fuchs for the release of detailed results of his relativistic Brueckner calculations in Ref. [65]. X.R., X.V., and M.C. acknowledge partial support by the Spanish Consolider-Ingenio 2010 Programme CPAN CSD2007-00042 and by Grants No. FIS2008-01661 from MICINN (Spain) and FEDER and No. 2009SGR-1289 from Generalitat de Catalunya (Spain). We also acknowledge partial support from the IN2P3CAICYT Collaboration (ACI10-00592) and from the DFG Cluster of Excellence "Origin and Structure of the Universe" (www.universe-cluster.de).
[1] D. Vautherin and D. M. Brink, Phys. Rev. C 5, 626 (1972).

[2] J. Dechargé and D. Gogny, Phys. Rev. C 21, 1568 (1980).

[3] P.-G. Reinhard, M. Rufa, J. Maruhn, W. Greiner, and J. Friedrich, Z. Phys. A 323, 13 (1986).

[4] G. A. Lalazissis, J. König, and P. Ring, Phys. Rev. C 55, 540 (1997).

[5] R. J. Furnstahl, B. D. Serot, and H.-B. Tang, Nucl. Phys. A 615, 441 (1997).

[6] S. Typel and H. H. Wolter, Nucl. Phys. A 656, 331 (1999).

[7] T. Nikšić, D. Vretenar, P. Finelli, and P. Ring, Phys. Rev. C 66, 024306 (2002).

[8] S. Goriely, M. Samyn, M. Bender, and J. M. Pearson, Phys. Rev. C 68, 054325 (2003).

[9] B. G. Todd-Rutel and J. Piekarewicz, Phys. Rev. Lett. 95, 122501 (2005).

[10] G. A. Lalazissis, T. Nikšić, D. Vretenar, and P. Ring, Phys. Rev. C 71, 024312 (2005).

[11] G. A. Lalazissis, S. Karatzikos, R. Fossion, D. Peña Arteaga, A. V. Afanasjev, and P. Ring, Phys. Lett. B 671, 36 (2009).

[12] M. Baldo, P. Schuck, and X. Viñas, Phys. Lett. B 663, 390 (2008).

[13] Extended Density Functionals in Nuclear Structure Physics, Vol. 641 of Lecture Notes in Physics, edited by G. A. Lalazissis, P. Ring, and D. Vretenar (Springer-Verlag, Heidelberg, 2004).

[14] J. E. Drut, R. J. Furnstahl, and L. Platter, Prog. Part. Nucl. Phys. 64, 120 (2010).

[15] P. Finelli, N. Kaiser, D. Vretenar, and W. Weise, Nucl. Phys. A 770, 1 (2006).

[16] T. Nikšić, D. Vretenar, and P. Ring, Phys. Rev. C 78, 034318 (2008).

[17] Universal Nuclear Energy Density Functional, SciDAC Collaboration [http://unedf.org/].

[18] S. Goriely, N. Chamel, and J. M. Pearson, Phys. Rev. C 82, 035804 (2010).

[19] M. Baldo, C. Maieron, P. Schuck, and X. Viñas, Nucl. Phys. A 736, 241 (2004).

[20] For a pedagogical introduction, see Nuclear Methods and the Nuclear Equation of State, edited by M. Baldo (World Scientific, Singapore, 1999).
[21] E. Garrido, P. Sarriguren, E. Moyade Guerra, and P. Schuck, Phys. Rev. C 60, 064312 (1999).

[22] L. M. Robledo, M. Baldo, P. Schuck, and X. Viñas, Phys. Rev. C 77, 051301 (2008).

[23] L. M. Robledo, M. Baldo, P. Schuck, and X. Viñas, Phys. Rev. C 81, 034315 (2010)

[24] M. Baldo, L. Robledo, P. Schuck, and X. Viñas, J. Phys. G 37, 064015 (2010).

[25] P.-G. Reinhard, Rep. Prog. Phys. 52, 439 (1989).

[26] P. Ring, Prog. Part. Nucl. Phys. 37, 193 (1996).

[27] B. D. Serot and J. D. Walecka, Int. J. Mod. Phys. E 6, 515 (1997).

[28] D. Vretenar, A. V. Afanasjev, G. A. Lalazissis, and P. Ring, Phys. Rep. 409, 101 (2005).

[29] J. Meng, H. Toki, S.-G. Zhou, S.-Q. Zhang, W.-H. Long, and L.-S. Geng, Prog. Part. Nucl. Phys. 57, 470 (2006).

[30] W. Koepf and P. Ring, Nucl. Phys. A 493, 61 (1989).

[31] A. V. Afanasjev and H. Abusara, Phys. Rev. C 81, 014309 (2010).

[32] U. Hofmann and P. Ring, Phys. Lett. B 214, 307 (1988).

[33] A. V. Afanasjev, P. Ring, and J. König, Nucl. Phys. A 676, 196 (2000).

[34] J. Dobaczewski and J. Dudek, Phys. Rev. C 52, 1827 (1995).

[35] H. P. Dürr and E. Teller, Phys. Rev. 101, 494 (1956).

[36] H. P. Dürr, Phys. Rev. 103, 469 (1956).

[37] J. D. Walecka, Ann. Phys. (NY) 83, 491 (1974).

[38] B. D. Serot and J. D. Walecka, Adv. Nucl. Phys. 16, 1 (1986).

[39] J. Boguta and A. R. Bodmer, Nucl. Phys. A 292, 413 (1977).

[40] W. H. Long, J. Meng, N. Van Giai, and S.-G. Zhou, Phys. Rev. C 69, 034319 (2004).

[41] R. Brockmann and H. Toki, Phys. Rev. Lett. 68, 3408 (1992).

[42] F. Hofmann, C. M. Keil, and H. Lenske, Phys. Rev. C 64, 034314 (2001).

[43] M. Serra, T. Otsuka, Y. Akaishi, P. Ring, and S. Hirose, Prog. Theor. Phys. 113, 1009 (2005).

[44] S. Hirose, M. Serra, P. Ring, T. Otsuka, and Y. Akaishi, Phys. Rev. C 75, 024301 (2007).

[45] W. Kohn and L. J. Sham, Phys. Rev. 137, A1697 (1965).

[46] W. Kohn and L. J. Sham, Phys. Rev. 140, A1133 (1965).

[47] P. Hohenberg and W. Kohn, Phys. Rev. 136, B864 (1964). 
[48] W. H. Long, N. Van Giai, and J. Meng, Phys. Lett. B 640, 150 (2006).

[49] W. H. Long, H. Sagawa, N. V. Giai, and J. Meng, Phys. Rev. C 76, 034314 (2007).

[50] W. H. Long, H. Sagawa, J. Meng, and N. Van Giai, Europhys. Lett. 83, 12001 (2008).

[51] W. H. Long, P. Ring, N. Van Giai, and J. Meng, Phys. Rev. C 81, 024308 (2010).

[52] H. Huber, F. Weber, and M. K. Weigel, Nucl. Phys. A 596, 684 (1996).

[53] H. Huber, F. Weber, and M. K. Weigel, Int. J. Mod. Phys. E 7, 301 (1998).

[54] S. Kubis and M. Kutschera, Phys. Lett. B 399, 191 (1997).

[55] J. Leja and Š. Gmuca, Acta Phys. Slov. 51, 201 (2001).

[56] B. Liu, V. Greco, V. Baran, M. Colonna, and M. DiToro, Phys. Rev. C 65, 045201 (2002).

[57] V. Greco, M. Colonna, M. DiToro, and F. Matera, Phys. Rev. C 67, 015203 (2003).

[58] S. S. Avancini, L. Brito, D. P. Menezes, and C. Providencia, Phys. Rev. C 70, 015203 (2004).

[59] T. Gaitanos, M. DiToro, S. Typel, V. Barana, C. Fuchs, V. Greco, and H. H. Wolter, Nucl. Phys. A 732, 24 (2004).

[60] V. Baran, M. Colonna, V. Greco, and M. DiToro, Phys. Rep. 410, 335 (2005).

[61] B. Liu, M. DiToro, and V. Greco, Int. J. Mod. Phys. E 9, 1815 (2008).

[62] H. Pais, A. Santos, and C. Providencia, Phys. Rev. C 80, 045808 (2009).

[63] A. Rabhi, C. Providencia, and J. DaProvidencia, Phys. Rev. C 80, 025806 (2009).

[64] G. B. Alaverdyan, Res. Astron. Astrophys. 10, 1255 (2010).

[65] E. N. E. van Dalen, C. Fuchs, and A. Faessler, Eur. Phys. J. A 31, 29 (2007).

[66] For a list of radioactive beam facilities in operation, under construction, or proposed, see [http://www.rarf.riken.go.jp/ rarf/np/nplab.html].

[67] H. Lenske and C. Fuchs, Phys. Lett. B 345, 355 (1995).

[68] C. Fuchs, H. Lenske, and H. H. Wolter, Phys. Rev. C 52, 3043 (1995).

[69] H. Liang, N. V. Giai, and J. Meng, Phys. Rev. C 79, 064316 (2009).

[70] J. D. Bjorken and S. D. Drell, Relativistic Quantum Fields (McGraw-Hill, New York, 1965).

[71] D. Vretenar, T. Nikšić, and P. Ring, Phys. Rev. C 68, 024310 (2003).

[72] R. Machleidt, Adv. Nucl. Phys. 19, 189 (1989).

[73] E. N. E. van Dalen, C. Fuchs, and A. Faessler, Phys. Rev. C 72, 065803 (2005).

[74] E. N. E. van Dalen, C. Fuchs, and A. Faessler, Phys. Rev. Lett. 95, 022302 (2005).

[75] T. Klähn et al., Phys. Rev. C 74, 035802 (2006).

[76] F. de Jong and H. Lenske, Phys. Rev. C 57, 3099 (1998).

[77] F. de Jong and H. Lenske, Phys. Rev. C 58, 890 (1998).

[78] M. Bender, K. Rutz, P.-G. Reinhard, and J. A. Maruhn, Eur. Phys. J. A 7, 467 (2000).

[79] D. Vautherin, Phys. Rev. C 7, 296 (1973).

[80] G. Audi, A. H. Wapstra, and C. Thibault, Nucl. Phys. A 729, 337 (2003).

[81] F. James, MINUIT-Function Minimization and Error Analysis (1998), CERN Program Library entry D506; Package Revision 1.1.1.1 (1996).
[82] I. Angeli, At. Data Nucl. Data Tables 87, 185 (2004).

[83] P. Danielewicz, R. Lacey, and W. G. Lynch, Science 298, 1592 (2002).

[84] B. A. Brown, Phys. Rev. Lett. 85, 5296 (2000).

[85] X. Roca-Maza, M. Centelles, X. Viñas, and M. Warda, Phys. Rev. Lett. 106, 252501 (2011).

[86] G. A. Souliotis, D. V. Shetty, A. Keksis, E. Bell, M. Jandel, M. Veselsky, and S. J. Yennello, Phys. Rev. C 73, 024606 (2006).

[87] D. V. Shetty, S. J. Yennello, and G. A. Souliotis, Phys. Rev. C 75, 034602 (2007).

[88] D. V. Shetty, S. J. Yennello, and G. A. Souliotis, Phys. Rev. C 76, 024606 (2007).

[89] A. W. Steiner and B.-A. Li, Phys. Rev. C 72, 041601(R) (2005).

[90] L.-W. Chen, C.-M. Ko, and B.-A. Li, Phys. Rev. Lett. 94, 032701 (2005).

[91] L.-W. Chen, C.-M. Ko, and B.-A. Li, Phys. Rev. C 72, 064309 (2005).

[92] L.-W. Chen, C.-M. Ko, and B.-A. Li, Phys. Rev. C 76, 054316 (2007).

[93] B.-A. Li, L.-W. Chen, and C.-M. Ko, Phys. Rep. 464, 113 (2008).

[94] P. Adrich et al., Phys. Rev. Lett. 95, 132501 (2005).

[95] A. Klimkiewicz et al., Phys. Rev. C 76, 051603(R) (2007).

[96] L. Trippa, G. Colò, and E. Vigezzi, Phys. Rev. C 77, 061304(R) (2008).

[97] M. Warda, X. Viñas, X. Roca-Maza, and M. Centelles, Phys. Rev. C 80, 024316 (2009).

[98] M. Centelles, X. Roca-Maza, X. Viñas, and M. Warda, Phys. Rev. Lett. 102, 122502 (2009).

[99] A. Trzcińska, J. Jastrzebski, P. Lubiński, F. J. Hartmann, R. Schmidt, T. von Egidy, and B. Klos, Phys. Rev. Lett. 87, 082501 (2001).

[100] B. Klos et al., Phys. Rev. C 76, 014311 (2007).

[101] J. Zenihiro et al., Phys. Rev. C 82, 044611 (2010).

[102] R. J. Furnstahl, B. D. Serot, and H.-B. Tang, Nucl. Phys. A 615, 441 (1997).

[103] G. Q. Li, R. Machleidt, and R. Brockmann, Phys. Rev. C 45, 2782 (1992).

[104] I. Vidana, C. Providencia, A. Polls, and A. Rios, Phys. Rev. C 80, 045806 (2009).

[105] G. A. Lalazissis (private communication).

[106] N. Tajima, P. Bonche, H. Flocard, P.-H. Heenen, and M. S. Weiss, Nucl. Phys. A 551, 434 (1993).

[107] M. M. Sharma, G. A. Lalazissis, and P. Ring, Phys. Lett. B 317, 9 (1993).

[108] M. M. Sharma, G. A. Lalazissis, J. König, and P. Ring, Phys. Rev. Lett. 74, 3744 (1995).

[109] P.-G. Reinhard and H. Flocard, Nucl. Phys. A 584, 467 (1995).

[110] E. W. Otten, in Radii Isotope Shifts by Laser Beam Spectroscopy, edited by D. A. Bromley (Plenum, New York, 1989), Vol. 8, p. 515

[111] W. Koepf and P. Ring, Z. Phys. A 339, 81 (1991).

[112] G. A. Lalazissis, D. Vretenar, and P. Ring, Phys. Rev. C 57, 2294 (1998)

[113] G. A. Lalazissis, D. Vretenar, W. Pöschl, and P. Ring, Phys. Lett. B 418, 7 (1998).

[114] S. Fayans, JETP Lett. 68, 169 (1998).

[115] T. D. Cohen, R. J. Furnstahl, and D. K. Griegel, Phys. Rev. C 45, 1881 (1992). 